1 2 3

\title{
Stiffness and strength of tridimensional periodic lattices
}

\author{
Andrea Vigliotti, Damiano Pasini* \\ Department of Mechanical Engineering, McGill University, 845 Sherbrooke St. W, \\ Montreal H3A 2T5, Canada
}

\begin{abstract}
This paper presents a method for the linear analysis of the stiffness and strength of open and closed cell lattices with arbitrary topology. The method hinges on a multiscale approach that separates the analysis of the lattice in two scales. At the macroscopic level, the lattice is considered as a uniform material; at the microscopic scale, on the other hand, the cell microstructure is modelled in detail by means of an in-house finite element solver. The method allows determine the macroscopic stiffness, the internal forces in the edges and walls of the lattice, as well as the global periodic buckling loads, along with their buckling modes. Four cube-based lattices and nine cell topologies derived by Archimedean polyhedra are studied. Several of them are characterized here for the first time with a particular attention on the role that the cell wall plays on the stiffness and strength properties. The method, automated in a computational routine, has been used to develop material property charts that help to gain insight into the performance of the lattices under investigation.
\end{abstract}

Keywords: lattice materials, periodic cellular materials, multiscale mechanics, stiffness and strength properties, periodic buckling, material property charts

\section{Introduction}

Lattices are regular structures obtained by tessellating a unit cell along independent periodic vectors. A lattice can be tailored by design to obtain

\footnotetext{
${ }^{*}$ Corresponding author

Email address: damiano.pasini@mcgill.ca (Damiano Pasini)
} 
unprecedented mechanical properties and access unexplored areas of the material property space (Banhart, 2001; Ashby, 2005; Fleck, 2010; Ashby, 2011). Recent manufacturing techniques enable to build complex lattice components at the micrometer length scale with high accuracy, acceptable costs (Bidanda and Bartolo, 2008; Ramirez, 2011), and from a variety of solid materials, including metal alloys, polymers, glass and silicon rubbers.

The microscopic characteristics of a lattice component govern its macroscopic behaviour. When the scale of the component is significantly larger than the scale of the lattice, a direct approach involving the modelling of each cell is impractical. This strategy would result in considerably large models, which are likely unfeasible to manage. On the other hand, an appropriate alternative is the substitution of the discrete model with an equivalent continuum (Noor, 1988). At the cost of loosing minor local details, this approach permits a substantial reduction of the computational effort, while still providing high accurate results.

In literature, there exist several methods to model the macroscopic properties of lattice materials. A force-based approach has been often applied to the unit cell of a lattice subjected to a multiaxial load. The elastic constants of the lattice have then been determined by solving each equilibrium problem individually. For example, Gibson et al. (1982) obtained a first order estimate of the in-plane stiffness of hexagonal honeycombs, by assuming the lattice edges behave as Bernoulli beams. Warren and Kraynik (1987) examined hexagonal lattices with non-constant edge thickness and modelled lattice edges as continua under plane stress. Zhu et al. (1997) and Gong et al. (2005) expressed the Young's modulus and the Poisson's ratio of open cell tetrakaidecahedral foams as a function of the relative density. For this cell topology, Ohno et al. (2010) derived the buckling and yielding strength under uniaxial compression. Among other cell topologies, pin-jointed fully triangulated lattices have attracted a remarkable interest for their excellent structural properties. Deshpande et al. (2001b) studied in detail the performance of the regular-octet lattice, and derived the lattice stiffness, and the von Mises surfaces for buckling and yielding. Elsayed (2010) applied shape transformers (Pasini, 2007) to study the effect of the edge cross section on the stiffness and strength of columns made out of octet lattices. Wallach and Gibson (2001) analysed a lattice based on tetrahedral units, and evaluated the effect of the cell aspect ratios on the stiffness, and on the buckling strength of alternative lattice. Wang and McDowell (2004) calculated the inplane stiffness and strength of a selected bidimensional cell topologies, with 
respect to the onset of plastic yielding.

Other works proposed a more general analysis of the mechanics of lattices based on topology optimization. Bendsoe and Kikuchi (1988), and later Hassani and Hinton (1998), for example, derived a constitutive model for porous materials considering an elementary unit cell of size $\epsilon$, with $\epsilon \rightarrow 0$. The virtual work principle was first applied to determine the deformation energy of the unit cell. The effective stiffness of the porous material was then obtained as the average, over the unit cell volume, of the stiffness of the solid material, weighted by the unit cell deformation energy.

The interest in vibration reduction and bandgaps has motivated the search for other approaches (Langley, 1996; Phani, 2006). The following can be loosely specified as procedural steps of these methods. (i) A displacement model is first proposed to approximate the displacements of the continuous medium within the unit cell; (ii) physical quantities of interest, such as natural frequency, stiffness, and strain energy, are calculated for the discrete lattice; (iii) the parameters of the continuous model are then determined by equating the relevant quantities of the discrete to those of the uniform model. While a Taylor series expansion is generally used to approximate the continuous displacement, the various methods might differ for the quantities to be equated in the models. For example, Kumar and McDowell (2004) selected the Cosserat description of the continuum to take into account the node rotations and the bending of the lattice elements. A second order Taylor series expansion was used for the displacements, and the coefficients of the equivalent micropolar continuum were found by comparing the expressions of the strain energy for the discrete lattice to those of the continuous medium. The suitability of the method was restricted to cell topologies with a single internal joint. Gonella and Ruzzene (2008) studied the in-plane properties of hexagonal and auxetic lattices and derived the parameters of the equivalent continuum by comparing the coefficients of the in-plane wave equations of a discrete lattice to those of an equivalent continuum model. Suiker et al. (2001) derived the parameters of a micropolar continuum considering the dispersion relations of harmonic waves. In both studies, a continuous model was used and provided a satisfactory approximation of the properties of the discrete lattice only for wavelengths longer than six times the dimension of the unit cell. For shorter wavelengths, local effects could not be captured. Gonella and Ruzzene (2010) recently observed that the use of the Taylor series approximation at short wavelength is the main culprit for the poor accuracy of the continuous model. Since it is not possible to increase the 
order of the expansion, which is limited by the number of boundary conditions that can be imposed, the authors proposed a representative volume element (RVE) made of multiple unit cells. Likewise with the aim of increasing the accuracy of the continuous model in capturing the local effects of wave propagation, Lombardo and Askes (2011) presented an approach based on a higher order approximation of the inertia terms only.

More recent works resorts to the application of concepts of crystals' theory. Hutchinson and Fleck (2006) proposed a method based on the Bloch theorem for the statement of the equilibrium problem over an infinite lattice, and relied on the Cauchy-Born assumption to interpolate the displacement in the unit cell. The method was applied to estimate the stiffness of the Kagome and the triangular-triangular lattice. The existence of inextensional periodic collapse modes was observed for the Kagome lattice, a stretching dominated material. Elsayed and Pasini (2010a) extended this approach to the analysis of more generic bidimensional stretching dominated lattices. Vigliotti and Pasini (2012) presented a matrix based method for the analysis of arbitrary bidimensional stretching and bending dominated lattices.

Works available in the literature are mainly focused on the evaluation of the lattice stiffness for pin-jointed open cell configurations. This paper presents a general procedure for the linear analysis of both open and closed cell three-dimensional lattices of arbitrary topology with either pin and rigid joints. The method hinges on a multiscale approach and makes no assumption on the displacements of the internal points of the unit cell; rather the change in the microscopic periodic directions is expressed as a function of the macroscopic strain filed, and the displacements of the deformed lattice are obtained by imposing equilibrium conditions. Besides stiffness, the procedure also permits the assessment of the strength of an arbitrary-cell lattice, with respect to both buckling and plastic yielding. Global buckling load and buckling modes are estimated by solving a generalized eigenvalue problem for the unit cell with prescribed periodic boundary conditions. The method is here applied to characterize the properties of 3D lattice topologies. Some topologies have been characterized here for the first time. In addition, since the method is based on the evaluation of the lattice strain energy by means of a finite element model of the unit cell, it can be extended to account for the effect of geometric and material non linearity. The method can also be applied recursively to analyse structure with multiple hierarchic levels of lattice structure.

The paper is structured as follows. First the multiscale approach is de- 
scribed in its general aspects in section 2 . In section 3 , the method for the lattice analysis is explained in detail. Section 4 applies the procedure for a comprehensive analysis of four lattice topologies based on the cubic unit cell. Here are given closed-form expressions of the stiffness and internal forces on lattice elements. The results of the analysis of the cubic-based topologies, and of nine lattices obtained from Archimedean solids, are finally used to develop material charts. A discussion comparing stiffness and strength properties of the lattices under investigation is given in section 5 , before the conclusion.

\section{The multiscale approach}

Structures built of lattice materials generally present at least two distinct length scales: the scale of the component, at the macroscopic level, and the scale of the unit cell, at the micro-level. Here, we formulate a comprehensive model consisting of two nested structural models. At the macroscopic level, the stiffness of the lattice component is determined by assuming the lattice material as a uniform continuum. At the microscopic level, we calculate the lattice stiffness and the internal forces in each lattice element, both essential for the analysis of the lattice strength.

The virtual work principle requires to equate the variation of the potential energy of the external forces to the variation of the strain energy, calculated through the constitutive relation of the material. For uniform materials, a functional relation exists between the stress and strain tensors and it reduces to the material stiffness matrix for the linear case. For lattice materials, since the relation depends on the properties of the lattice, it cannot be expressed directly as a functional dependence. Yet, a boundary value problem can be formulated to calculate the lattice strain energy and to express it as a function of the macroscopic strain. Figure 1 summarizes the steps of the

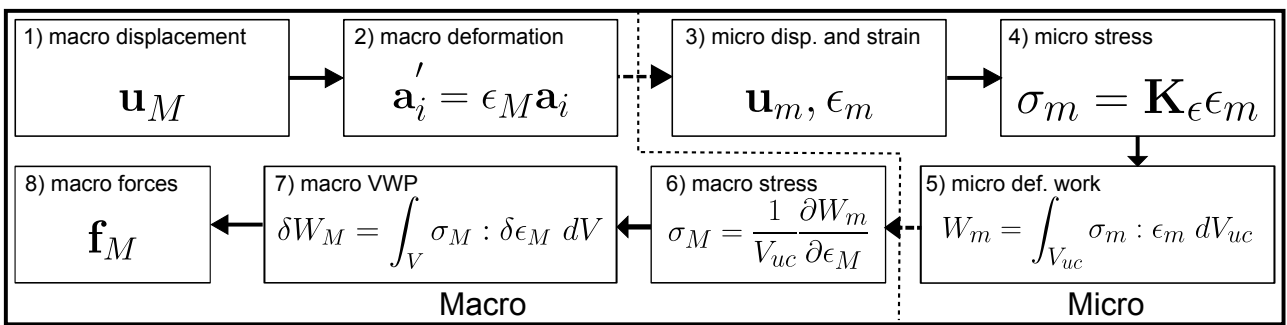

Figure 1: Multiscale scheme

multiscale scheme, which is largely based on the work by Kouznetsova et al. 
(2002). At the component level $(1,2)$, given the macroscopic displacements, $\mathbf{u}_{M}$, we determine the components of the Cauchy strain tensor, $\epsilon_{M}$, and the deformed lattice periodic vectors, $\mathbf{a}_{i}$; the micro-displacements of the unit cell nodes are expressed as a function of the macroscopic strain (3); the microstress of the uniform solid material is obtained via the Hooke's law (4); the micro-deformation work is calculated by means of a finite element model of the unit cell (5); the macro-stress tensor is calculated as the gradient of the strain energy density with respect to the macroscopic strain (6); macroforces are obtained applying the virtual work principle at the macroscopic level $(7,8)$.

Two conditions are imposed to define the equilibrium problem of the unit cell: a kinematic condition to preserve the periodicity of the unit cell boundaries; a static condition to ensure the equilibrium of the confining cells. The formulation enables the analysis of a lattice with arbitrary cell topology and any number of either pin or rigid joints both on the boundary and inner of the unit cell. Once the equilibrium of the unit cell has been solved, the deformation work and the forces in the cell elements can be determined as a function of the macroscopic strain tensor.

The components of the macroscopic stress field can be obtained by applying the virtual work principle on the unit cell. Equating the virtual work at the micro- with that at the macro- scale, and recalling that the macroscopic quantities are assumed uniform over the unit cell, we obtain

$$
\delta W=\int_{V_{u c}} \sigma_{m}: \delta \epsilon_{m} d V_{u c}=V_{u c} \sigma_{M}: \delta \epsilon_{M}
$$

where $V_{u c}$ is the volume of the unit cell. The virtual variation of the deformation work can also be expressed as $\delta W=\frac{\partial W}{\partial \epsilon_{M}} \delta \epsilon_{M}$; hence the macroscopic stress tensor can be obtained as

$$
\sigma_{M}=\frac{1}{V_{u c}} \frac{\partial W}{\partial \epsilon_{M}}
$$

The method is based on a finite element model of the unit cell for the calculation of the deformation work. Since this paper focuses on a linear analysis, the lattice stiffness matrix, $\mathbf{K}_{\epsilon}$, can be calculated as the Hessian of the deformation work with respect to components of the macroscopic deformation tensor. 


\section{Analysis of three-dimensional periodic lattices}

In this section, we apply periodic boundary conditions to derive the properties of the infinite lattice as a function of the properties of a single cell. We start with the kinematic constraints applied at the nodes of the cell boundaries, which must guarantee the lattice periodicity.

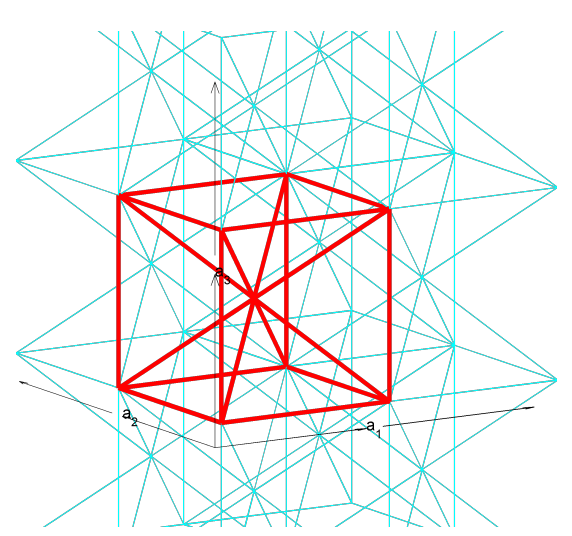

(a) replicated cell

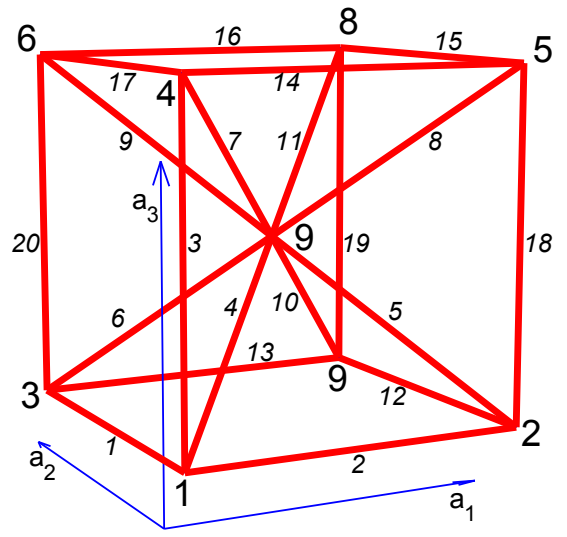

(b) unit cell

Figure 2: Body centred cubic cell

The position $\mathbf{r}_{k}$ of a given node, edge, wall, and any other entity of the infinite lattice, is linked to the position $\mathbf{r}_{0}$ of the same entity in the reference unit cell through the periodic vectors, $\mathbf{a}_{i}$, as shown in Figure $2 \mathrm{a}$ for the Body Centred Cubic lattice. Hence $\mathbf{r}_{k}$ can be expressed as

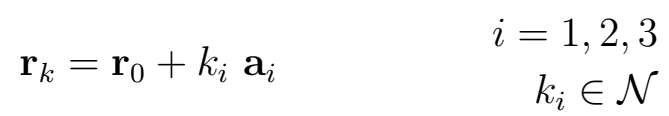

Under a uniform macroscopic stress, an infinite lattice deforms while maintaining its periodicity. If $\mathbf{a}_{i}$ are the deformed periodic vectors, the position of the lattice entities in the deformed configuration, and their displacements, $\mathbf{u}_{k}$, can be written as

$$
\mathbf{r}_{k}^{\prime}=\mathbf{r}_{0}^{\prime}+k_{i} \mathbf{a}_{i}^{\prime}
$$

The nodal displacement can be obtained subtracting equation (4) from equation (3), which yields the following

$$
\mathbf{u}_{k}=\mathbf{u}_{0}+k_{i} \Delta \mathbf{a}_{i}
$$


where $\Delta \mathbf{a}_{i}$ is the change of the $i-t h$ periodic direction, and $\mathbf{u}_{0}$ is the displacement of the point $\mathbf{r}_{0}$.

With reference to the nodes of a single cell, we can identify two separate classes of nodes: the internal nodes connecting elements of the same unit cell, and the boundary nodes that connect elements of the confining cells. Due to periodicity, we observe that the boundary nodes must correspond along the periodic vectors. Hence, a subset of the unit cell nodes is sufficient to generate all the nodes of the lattice. These independent nodes comprise the internal nodes, which have no corresponding node in the unit cell, and a selection of the boundary nodes. Through equation (5), the displacements of all the nodes of the lattice can be expressed as a function of the displacements of the independent nodes.

With reference to Figure 2b, we can verify that node 9 is the only internal node; all other nodes are boundary nodes and any of them can be considered as the independent node. In particular, selecting select node 1 as independent node, the following relations hold

$$
\begin{array}{lll}
\mathbf{r}_{2}=\mathbf{r}_{1}+\mathbf{a}_{1} & \mathbf{r}_{3}=\mathbf{r}_{1}+\mathbf{a}_{2} & \mathbf{r}_{4}=\mathbf{r}_{1}+\mathbf{a}_{3} \\
\mathbf{r}_{5}=\mathbf{r}_{1}+\mathbf{a}_{1}+\mathbf{a}_{3} & \mathbf{r}_{6}=\mathbf{r}_{1}+\mathbf{a}_{2}+\mathbf{a}_{3} & \mathbf{r}_{7}=\mathbf{r}_{1}+\mathbf{a}_{1}+\mathbf{a}_{2} \\
\mathbf{r}_{8}=\mathbf{r}_{1}+\mathbf{a}_{1}+\mathbf{a}_{2}+\mathbf{a}_{3} & &
\end{array}
$$

Further details on the derivation of equations (3) and (5) for a generic lattice are given in Appendix A.

In the following, we assume that a finite element model of the unit cell is available and each node has 6 DoFs. Hence, the status of the cell can be fully described by the array of nodal DoFs, $\mathbf{d}$. The DoFs of the unit cell can be expressed as a function of $\mathbf{d}_{0}$, the array of the DoFs of the independent nodes, and of the change in the periodic directions, through the equation

$$
\mathbf{d}=\mathbf{B}_{0} \mathbf{d}_{0}+\mathbf{B}_{\mathrm{a}} \Delta \mathbf{a}
$$

where the array $\Delta \mathbf{a}$ collects the components of the $\Delta \mathbf{a}_{i}$ vectors, and $\mathbf{B}_{0}$ and $\mathbf{B}_{\mathrm{a}}$ are block matrices which depend of the lattice topology. The derivation and properties of the matrices $\mathbf{B}_{0}$ and $\mathbf{B}_{\mathrm{a}}$ are explained in Appendix $\mathrm{A}$.

We now consider the static equilibrium of the infinite lattice. In the deformed configuration, the internal forces of any cell of the lattice must balance the forces applied by the surrounding cells. In the linear case, the nodal forces of the unit cell can be expressed as

$$
\mathbf{F}=\mathbf{K}_{\mathrm{uc}} \mathbf{d}
$$


where $\mathbf{K}_{\mathrm{uc}}$ is the unit cell stiffness matrix. As described in Appendix A, the periodic equilibrium conditions can be expressed in a compact form as

$$
\mathbf{A}_{0} \mathbf{F}=\mathbf{A}_{0} \mathbf{K}_{\mathrm{uc}} \mathbf{d}=\mathbf{0}
$$

where $\mathbf{A}_{0}$ is a block matrix, such that $\mathbf{A}_{0}=\mathbf{B}_{0}^{T}$. Combining equations (9) and (7), and separating the term in $\mathbf{d}_{0}$ from the term in $\boldsymbol{\Delta} \mathbf{a}$, we obtain the following equation for the independent DoFs

$$
\mathbf{B}_{0}^{T} \mathbf{K}_{\mathrm{uc}} \mathbf{B}_{0} \mathbf{d}_{0}=-\mathbf{B}_{0}^{T} \mathbf{K}_{\mathrm{uc}} \mathbf{B}_{\mathrm{a}} \boldsymbol{\Delta} \mathbf{a}
$$

We observe that the left hand side of equation (10) represents the unbalance forces due to $\mathbf{d}_{0}$, if the periodic vectors are kept fixed. In contrast, the right hand side is the force unbalance due to a variation of the periodic vectors, if the independent DoFs are zero. Thus, the solution of equation (10) is the array of the independent DoFs of the unit cell that equilibrates the forces due to surrounding cells, originated by the lattice deformation; and the matrix $\mathbf{B}_{0}^{T} \mathbf{K}_{\mathrm{uc}} \mathbf{B}_{0}$ effectively represents the constrained stiffness matrix of the unit cell.

Since both sides of equation (10) belong to the column space of the matrix $\mathbf{B}_{0}^{T}$, non trivial solutions will always exist. Being $\mathbf{K}_{\mathrm{uc}}$ the stiffness matrix of the unconstrained unit cell, its null space is not empty, and so is the null space of $\mathbf{B}_{0}^{T} \mathbf{K}_{\mathrm{uc}} \mathbf{B}_{0}$. Hence, the solution of (10) is not unique; rather it represents an affine space (Strang, 2006). A particular solution is given by the equation (11), where ${ }^{+}$denotes the Moore-Penrose pseudo-inverse.

$$
\mathbf{d}_{0}=-\left(\mathbf{B}_{0}^{T} \mathbf{K}_{\mathrm{uc}} \mathbf{B}_{0}\right)^{+} \mathbf{B}_{0}^{T} \mathbf{K}_{\mathrm{uc}} \mathbf{B}_{\mathrm{a}} \boldsymbol{\Delta} \mathbf{a}=\mathbf{D}_{0} \boldsymbol{\Delta} \mathbf{a}
$$

Combining equations (7) and (11), we obtain the generalized displacements of the unit cell nodes, $\mathbf{d}$, that satisfy both the equilibrium condition, and the displacement periodicity requirement, as a function of the change in the periodic vectors, $\Delta \mathbf{a}$,

$$
\mathbf{d}=\left(\mathbf{B}_{0} \mathbf{D}_{0}+\mathbf{B}_{\mathrm{a}}\right) \Delta \mathbf{a}=\mathbf{D}_{\mathrm{a}} \Delta \mathbf{a}
$$

Equation (12) can be used to evaluate the deformation work of the unit cell as a function of the change in the periodic vectors, as follows

$$
W=\frac{1}{2} \mathbf{d}^{T} \mathbf{K}_{\mathrm{uc}} \mathbf{d}=\frac{1}{2} \boldsymbol{\Delta} \mathbf{a}^{T} \mathbf{D}_{\mathrm{a}}^{T} \mathbf{K}_{\mathrm{uc}} \mathbf{D}_{\mathrm{a}} \boldsymbol{\Delta} \mathbf{a}=\frac{1}{2} \boldsymbol{\Delta} \mathbf{a}^{T} \mathbf{K}_{\Delta \mathrm{a}} \boldsymbol{\Delta} \mathbf{a}
$$


As it was noted earlier, the solution to equation (10) is an affine space. Nevertheless, since the columns of $\mathbf{B}_{0}$ are independent, the members of $\operatorname{Null}\left(\mathbf{B}_{0}^{T} \mathbf{K}_{\mathrm{uc}} \mathbf{B}_{0}\right)$ do not produce any mechanical work, it follows that expression (13) is unique with respect to the deformation work. In other words, all the elements of the affine space, i.e. the solutions of equation (10), produce the same unit cell deformation work, and lead to the same expression of the lattice stiffness.

If we examine the eigenvalues and eigenvectors of $\mathbf{K}_{\Delta a}$ in the tridimensional case, we note that $\mathbf{K}_{\Delta \mathrm{a}}$ has dimensions $9 \times 9$. Three of the nine eigenvalues are zero as they correspond to rigid lattice rotations. If additional null eigenvalues appear, they correspond to mechanisms that might be present in the lattice. The eigenvectors corresponding to the largest eigenvalue represent the deformation modes for which the lattice offers the highest stiffness. Thus along these load directions, the structural performance of the lattice is maximum. On the other hand, the eigenvectors related to the smallest non-zero eigenvalue correspond to load conditions for which the lattice has maximum compliance. If the lattice has to withstand a multiaxial load, it will exhibit the largest deformation, and eventually fail, along these directions.

\subsection{Strain-displacement model and lattice stiffness}

In this section, the deformation work is first derived as a function of the components of a uniform macroscopic strain field acting on the lattice; then it is used to obtain the lattice macroscopic stiffness. From the definition of the Cauchy strain tensor (Asaro and Lubarda, 2006), the change in the periodic vectors can be related to the macroscopic strain field as follows

$$
\mathbf{a}^{\prime}=\left(\mathbf{I}+\epsilon_{M}\right) \mathbf{a} \Rightarrow \Delta \mathbf{a}=\epsilon_{M} \mathbf{a}
$$

where $\epsilon_{M}$ is the Cauchy strain tensor. For the three dimensional case, the components of $\boldsymbol{\Delta} \mathbf{a}$ are given by

$$
\left[\begin{array}{c}
\Delta a_{x} \\
\Delta a_{y} \\
\Delta a_{z}
\end{array}\right]=\left[\begin{array}{ccc}
\epsilon_{x} & \epsilon_{x y} & \epsilon_{x z} \\
\epsilon_{x y} & \epsilon_{y} & \epsilon_{y z} \\
\epsilon_{z x} & \epsilon_{y z} & \epsilon_{z}
\end{array}\right]\left[\begin{array}{l}
a_{x} \\
a_{y} \\
a_{z}
\end{array}\right]
$$

the above equation can be rearranged, and expressed in terms of the components of the macroscopic engineering strain field, as

$$
\left[\begin{array}{c}
\Delta a_{x} \\
\Delta a_{y} \\
\Delta a_{z}
\end{array}\right]=\left[\begin{array}{cccccc}
a_{x} & 0 & 0 & \frac{a_{y}}{2} & 0 & \frac{a_{z}}{2} \\
0 & a_{y} & 0 & \frac{a_{x}}{2} & \frac{a_{z}}{2} & 0 \\
0 & 0 & a_{z} & 0 & \frac{a_{y}}{2} & \frac{a_{x}}{2}
\end{array}\right] \quad\left[\begin{array}{c}
\epsilon_{x} \\
\epsilon_{y} \\
\epsilon_{z} \\
\gamma_{y z} \\
\gamma_{z x} \\
\gamma_{x y}
\end{array}\right]
$$


where $\gamma_{i j}=2 \epsilon_{i j}$. The equations for all periodic vectors can be grouped, in a compact form, in one array as

$$
\Delta \mathbf{a}=\mathbf{B}_{\epsilon} \epsilon_{M} \quad \text { with } \quad \mathbf{B}_{\epsilon}=\left[\begin{array}{cccccc}
a_{1 x} & 0 & 0 & \frac{a_{1 y}}{2} & 0 & \frac{a_{1 z}}{2} \\
0 & a_{1 y} & 0 & \frac{a_{1 x}}{2} & \frac{a_{1 z}}{2} & 0 \\
0 & 0 & a_{1 z} & 0 & \frac{a_{1 y}}{2} & \frac{a_{1 x}}{2} \\
a_{2 x} & 0 & 0 & \frac{a_{2 y}}{2} & 0 & \frac{a_{2 z}}{2} \\
0 & a_{2 y} & 0 & \frac{a_{2 x}}{2} & \frac{a_{2 z}}{2} & 0 \\
0 & 0 & a_{2 z} & 0 & \frac{a_{2 y}}{2} & \frac{a_{2 x}}{2} \\
a_{3 x} & 0 & 0 & \frac{a_{3 y}}{a_{2}} & 0 & \frac{a_{3 z}}{2} \\
0 & a_{3 y} & 0 & \frac{33 x}{2} & \frac{a_{3 z}}{2} & 0 \\
0 & 0 & a_{3 z} & 0 & \frac{a_{3 y}}{2} & \frac{a_{3 x}}{2}
\end{array}\right]
$$

Substituting equation (17) into equation (12) and (13), allows to express the unit cell nodal DoFs, and the unit cell deformation work as a function of the components of the macroscopic strain field, as

$$
\begin{aligned}
& \mathbf{d}=\mathbf{D}_{\mathrm{a}} \mathbf{B}_{\epsilon} \epsilon_{M}=\mathbf{D}_{\epsilon} \epsilon_{M} \\
& W=\frac{1}{2} \epsilon_{M}^{T} \mathbf{B}_{\epsilon}^{T} \mathbf{K}_{\Delta \mathrm{a}} \mathbf{B}_{\epsilon} \epsilon_{M}
\end{aligned}
$$

The expression for the lattice macroscopic stiffness can be derived from equation (19); since the material stiffness is equal to the Hessian of the strain energy with respect to the deformation components, $K_{i j h k}=\frac{\partial^{2} W}{\partial \epsilon_{i j} \partial \epsilon_{h k}}$, we can write the following expression

$$
\mathbf{K}_{\epsilon}=\frac{1}{V} \mathbf{B}_{\epsilon}^{T} \mathbf{K}_{\Delta \mathrm{a}} \mathbf{B}_{\epsilon} \quad \text { with } \quad V=\left|\mathbf{a}_{1} \cdot \mathbf{a}_{2} \times \mathbf{a}_{3}\right|
$$

where $V$ is the volume of the unit cell. Because the change in the periodic vectors is now expressed in terms of the components of the macroscopic strain field, $\mathbf{K}_{\epsilon}$ does not include any rigid rotation mode; nevertheless, zero eigenvalues can still exist if the unit cell holds a mechanism, such as in the case of pin-jointed statically indeterminate lattices.

\subsection{Determination of the internal forces}

The multiscale approach presented in this paper can be used to determine the stress and strain field in the lattice elements as a function of an applied macroscopic stress or strain field. When a macroscopic stress field is acting on the lattice, the resulting internal forces in the lattice elements can lead to either a microscopic plastic yielding or buckling. To assess the lattice 
strength, the effective buckling load and the buckling modes of the lattice elements should be calculated as a function of the macroscopic stress, and of the lattice parameters.

When a lattice is modelled as a continuum by means of its equivalent stiffness, the result of the analysis is a macroscopic stress field. To assess the lattice strength, the macroscopic stress field of the continuum should be expressed in terms of the stress of the solid material of the lattice. The components of the microscopic stress and strain tensors can be calculated by the steps from 1 to 4 of the multiscale scheme illustrated in Figure 1. In the linear case, these steps are grouped in the array $\mathbf{D}_{\epsilon}$ of equation (18) which expressed the components of the macroscopic strain as a function of the DoFs of the unit cell joints. The DoFs of the unit cell can be expressed in terms of the macro-stress as follows

$$
\mathbf{d}=\mathbf{D}_{\epsilon} \mathbf{C}_{\epsilon} \sigma_{M}
$$

where $\mathbf{C}_{\epsilon}=\mathbf{K}_{\epsilon}^{-1}$, is the lattice compliance matrix. Given the DoFs of the unit cell nodes, $\mathbf{K}_{\epsilon}$ can be used to calculate the forces in the lattice elements. For instance, if the cell edges are modelled as Euler-Bernoulli beams, linear shape functions are assumed for the stretching and torsion, and cubic functions are assumed for the bending, the following holds (Zienkiewicz and Taylor, 2005)

$$
\begin{aligned}
& s=\frac{u_{2}-u_{1}}{L} \\
& \chi_{y}=x\left(12 \frac{w_{1}-w_{2}}{L^{3}}-6 \frac{\theta_{y 1}+\theta_{y 2}}{L^{2}}\right)+6 \frac{w_{2}-w_{1}}{L^{2}}+2 \frac{2 \theta_{y 1}+\theta_{y 2}}{L} \\
& \chi_{z}=x\left(12 \frac{v_{1}-v_{2}}{L^{3}}+6 \frac{\theta_{z 1}+\theta_{z 2}}{L^{2}}\right)+6 \frac{v_{2}-v_{1}}{L^{2}}-2 \frac{2 \theta_{z 1}+\theta_{z 2}}{L} \\
& \phi=\theta_{x 2}-\theta_{x 1}
\end{aligned}
$$

where $x$ varying from 0 to $L$ is the abscissa along the element, $s$ is the edge stretching, $\chi_{i}$ is the curvature around the $-i$ axis, $\phi$ is the angle of twist. The components of the nodal DoFs are specified in Figure 3. The normal force, the bending moments and the torsion moments are, thus, given by the following

$$
\begin{aligned}
& N=E_{s} A s \\
& M_{y}=E_{s} I_{z z} \chi_{y} \\
& M_{z}=E_{s} I_{y y} \chi_{z} \\
& T=\frac{G_{s} J_{p}}{L} \phi
\end{aligned}
$$

where $E_{s}$ and $G_{s}$ are the Young's and the shear moduli of the solid material, $A$ and $I_{i i}$ are the cross section area and the second moments of area with respect to the $-i$ axis, and $J_{p}$ is the polar moment of inertia. 


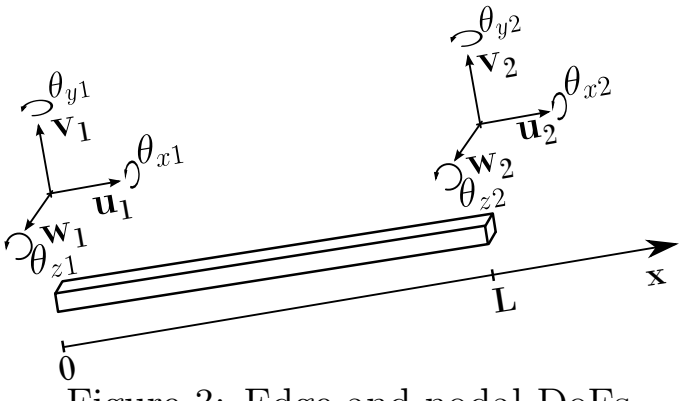

Figure 3: Edge and nodal DoFs

\subsection{Lattice strength and periodic buckling}

The occurrence of local buckling should be verified if the macroscopic stress produces compression in a lattice element. Deshpande et al. (2001b) calculated the buckling strength of the pin-jointed regular octet with open cell. If the lattice is rigid-jointed, this assumption yields an underestimation of the actual buckling load. A given joint stiffness can delay, or even prevent, the occurrence of a buckling mode, especially if there are cell walls that stiffen the cell edges along their length. Thus, to estimate accurately the buckling load resistance, we need to formulate the generalized eigenvalue problem, including the periodic boundary conditions, as described in the previous section.

The buckling loads and buckling modes for a structure can be found by solving the following (Cook et al., 1989).

$$
\left(\mathbf{K}+\lambda \mathbf{K}_{\sigma}\right) \mathbf{x}=0
$$

where $\mathbf{K}$ and $\mathbf{K}_{\sigma}$ are the stiffness matrix and the stress stiffness matrix of the structure. To order to calculate the global lattice critical loads, we need to take into account the effective loads on a single cell and its constraints induced by the surrounding cells. For a given macroscopic stress, the stress stiffness matrix can be obtained by imposing node displacements, as given by equation (20). Since the matrix $\mathbf{B}_{0}$, in equation (21), introduces periodic boundary conditions on the unit cell, $\mathbf{B}_{0}^{T} \mathbf{K}_{u c} \mathbf{B}_{0}$ represents the constrained stiffness matrix of the unit cell. It follows that buckling loads and modes of the lattice can be obtained by solving the following

$$
\left(\mathbf{B}_{0}^{T} \mathbf{K}_{u c} \mathbf{B}_{0}+\lambda \mathbf{B}_{0}^{T} \mathbf{K}_{u c_{\sigma}} \mathbf{B}_{0}\right) \mathbf{d}_{0}=0
$$

where $\mathbf{K}_{u c_{\sigma}}$ is the unconstrained stress stiffness matrix of the unit cell. For a prescribed macroscopic stress field, the corresponding stress stiffness 
matrix is first obtained and, through equation (25), the eigenvalues and eigenvectors pairs corresponding to the buckling loads and modes can be determined. The smallest eigenvalue, $\lambda_{c r}$, is the multiplying factor for the applied stress that triggers buckling on the cell elements. Thus, if $\lambda_{c r}=1$, buckling occurs. The relative eigenvector, $\mathbf{d}_{0_{c r}}$, contains the independent DoFs of the buckling mode; the DoFs of all nodes of the unit cell can then be obtained via equation (7). Figure 4 shows the first buckling modes, corresponding to three distinct stress states, for the Body Centred Cube (BCC) topology.

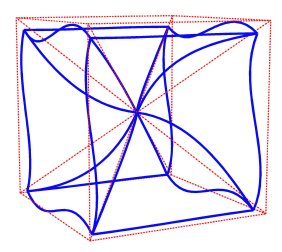

(a) Hydrostatic

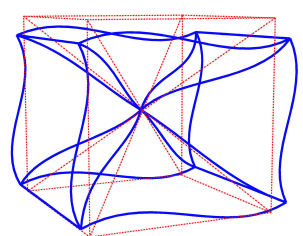

(b) Bending

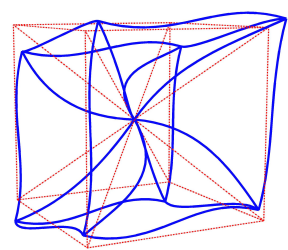

(c) Shear

Figure 4: BCC topology, buckling modes

\section{Analysis of selected topologies}

The procedure described in the paper is applied to determine the properties of 13 different lattices. We consider here four cell topologies derived from the regular cubic topology (Figure 5), and nine based on the tessellation of a selection of Archimedean polyhedra (Table 6). Since all cells have cubic symmetry, there exist nine mutually orthogonal planes of symmetry, and the stiffness matrix can be written, in a reference system with the axes defined by intersection of the symmetry planes (Asaro and Lubarda, 2006), as

$$
\mathbf{K}_{\text {mat }}=\left[\begin{array}{cccccc}
\alpha & \beta & \beta & 0 & 0 & 0 \\
\beta & \alpha & \beta & 0 & 0 & 0 \\
\beta & \beta & \alpha & 0 & 0 & 0 \\
0 & 0 & 0 & \gamma & 0 & 0 \\
0 & 0 & 0 & 0 & \gamma & 0 \\
0 & 0 & 0 & 0 & 0 & \gamma
\end{array}\right]
$$

For an isotropic material, the terms of the material stiffness matrix depend on two independent parameters only (the Young's Modulus, $E_{s}$, and the Poisson's ratio, $\nu$ ). On the other hand, for the lattice materials under investigation $\alpha, \beta$ and $\gamma$ are independent. Yet, the stiffness matrix of a uniform material has identical eigenvectors, as reported in Table 1. Hence, to compare the lattice stiffness to the stiffness of a solid material, we can compare 


\begin{tabular}{|l|l|l|}
\hline Solid material & Lattice & Eigenvector \\
\hline$\lambda_{1 s}=\frac{E_{s}}{1-2 \nu}$ & $\lambda_{h}=\alpha+2 \beta$ & $\epsilon_{h}=[1,1,1,0,0,0] \frac{1}{\sqrt{3}}$ \\
\hline$\lambda_{2 s}=\frac{E_{s}}{\nu+1}$ & $\lambda_{d}=\alpha-\beta$ & $\epsilon_{d 1}=[1,-1,0,0,0,0] \frac{1}{\sqrt{2}}$ \\
& & $\epsilon_{d 2}=[1,0,-1,0,0,0] \frac{1}{\sqrt{2}}$ \\
\hline \multirow{3}{*}{$\lambda_{3 s}=\frac{E_{s}}{2(\nu+1)}$} & $\lambda_{s}=\gamma$ & $\epsilon_{s 1}=[0,0,0,1,0,0]$ \\
& & $\epsilon_{s 2}=[0,0,0,0,1,0]$ \\
& $\epsilon_{s 3}=[0,0,0,0,0,1]$ \\
\hline
\end{tabular}

Table 1: Eigenvalues and eigenvectors for the selected topologies obtained from $\mathbf{K}_{\text {mat }}$

the eigenvalues of the same eigenvectors. The first eigenvalue, $\lambda_{h}$ corresponds to the pure hydrostatic stress state; its algebraic multiplicity is three, while the associate eigenspace is monodimensional. Usually, it is the largest. The second eigenvalue, $\lambda_{d}$, has both algebraic and geometrical multiplicity two and defines the deviatoric stress states. The third eigenvalue is determined by pure macroscopic shear stress, has geometrical multiplicity three and is generally the smallest. A characteristic property of lattice materials is the deformation behaviour, which can be bending or stretching dominated (Deshpande et al., 2001a). Stretching dominated lattices basically respond with element stretching to any macroscopic load, whereas for bending dominated, under some loading conditions, the lattice elements essentially flex. In the latter case, the stiffness of the lattice is significantly smaller, or even null for pin jointed connections. It follows that for a stretching dominated lattice, all the eigenvalues of the stiffness matrix have the same order of magnitude, and scale with $\rho^{*}$. On the other hand, for bending dominated lattices, the modes that are absorbed as bending correspond to smaller eigenvalues, which scale significantly faster to zero, as the power law is controlled by $\rho^{*^{2}}$.

\subsection{Finite element modelling of the unit cells}

The numerical results presented in this paper have been obtained by means of in-house finite element scripts. The lattice edges have been modelled as Euler Bernoulli beams neglecting shear. When not triangular, the walls have been meshed with triangular elements, adding a node in the centre of the face. The in-plane behaviour of the walls has been modelled with first order plane stress elements, while the bending behaviour has been modelled as BCIZ plates Bazeley et al. (1965), to ensure an exact representation of each 
constant curvature mode. This aspect eases the evaluation of the buckling loads in the edges. Since the first beam buckling load has constant curvature, the stiffening effect of the adjacent walls can be calculated accurately.

With respect to the cell geometry, the thickness $t$ of the cell walls is assumed constant. If we then define $L$ as the length of the selected edges and $d$ as the side of edges cross section, which is considered square, then the lattice geometry can be readily normalized and expressed with respect to the dimensionless parameters

$$
\begin{array}{lc}
l_{1}=\frac{L}{d} & 10 \leq l_{1} \leq 50 \\
l_{2}=\frac{t}{d} & 0 \leq l_{2} \leq \frac{1}{4}
\end{array}
$$

where $l_{1}$ is the slenderness ratio of the edges, and $l_{2}$ is the ratio of the wall thickness with respect to the edge cross section side. The bounds on $l_{1}$ derives from the assumption of slender beams, while the bound on $l_{2}$ is necessary to avoid the overestimation of mass and of joint stiffness.

\subsection{Construction of the lattice property charts}

Material property charts (Ashby, 2005) are a visual mean to compare material performance. In this section, the properties of the lattices are plotted into material charts. Each map shows the attainable range of the stiffness and strength of each lattice as a function of the relative density. Since the lattice parameters are controlled by the ratios $l_{1}$ and $l_{2}$, the design space of the lattice is represented as a region of the plane. Figure 7 shows a contour plot of the relative density of the cubic lattice as a function of $l_{1}$ and $l_{2}$; the material charts are obtained by calculating the range of the values of each property on lines with constant relative density.

In the next sections, the symbolic expressions of the stiffness matrix entries, given for the cubic-based topologies, are used to plot stiffness and strength properties. The ordinates of each chart have been normalized to produce dimensionless plots. With reference to yield, the lattice strength is controlled by the yield of the solid material. For a given applied macroscopic stress, the maximum von Mises stress of the cell has been compared to the yield of the solid material, which is assumed linear elastic and perfectly plastic, with a proportional limit of $\epsilon_{L}=0.01$. With respect to the lattice buckling strength, the first critical load of the edges has been considered as representative of the lattice global strength. This choice stems from the 
observation that the critical load on the walls is very small and the edges provide a considerable strength.

\subsection{Cubic topologies}

This section focuses on the analysis of four lattice topologies obtained from a cubic envelope. The cell symmetry yields to simplified closed-form expressions for the entries of the lattice stiffness matrix. The properties are found in a reference system with axes aligned with the sides of the base cube, see Figure 2b. Due to the linearity assumptions, the bending and the stretching contribution to the material stiffness can be determined separately. Thus the weight of each contribution can be expressed with respect to the overall lattice stiffness.

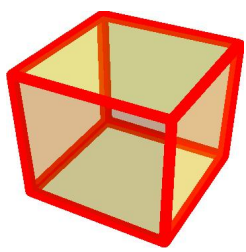

(a) Regular cubic

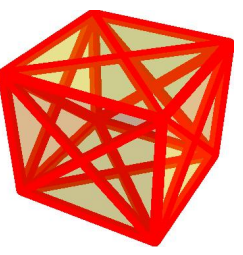

(b) FCC

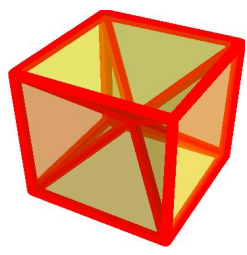

(c) $\mathrm{BCC}$

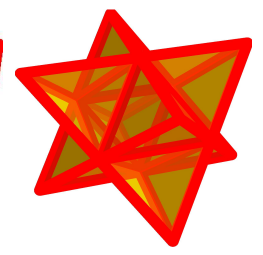

(d) Regular Octet

Figure 5: Cubic topologies

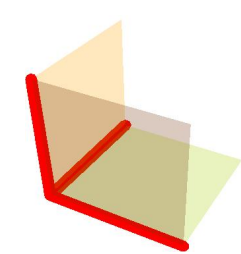

(a) Regular cubic

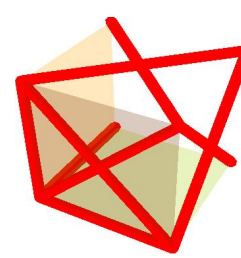

(b) FCC

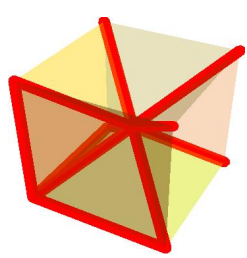

(c) $\mathrm{BCC}$

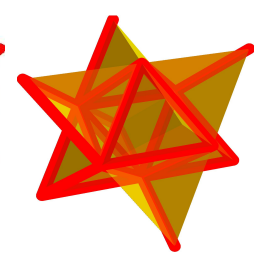

(d) Regular Octet

Figure 6: Primitive cells of cubic cell topologies, Figure 5

\subsubsection{Regular cube}

Figure 5a shows the regular cubic topology. Its primitive cell consists of three edges and three walls (Figure 6a) from which the infinite lattice can be generated. For the primitive cubic topology, the linear expression of the relative density is given by

$$
\rho^{*}=3\left(\frac{A}{L^{2}}+\frac{t}{L}\right)
$$




\begin{tabular}{|c|c|c|c|c|}
\hline \multirow{2}{*}{} & \multicolumn{2}{|c|}{ Edges } & \multicolumn{2}{c|}{ Walls } \\
\cline { 2 - 5 } & Axial & Bending & In-plane & Bending \\
\hline$\frac{\alpha}{E_{s}}$ & $\frac{A}{L^{2}}$ & 0 & $\frac{t}{L} \frac{2}{1-\nu^{2}}$ & 0 \\
\hline$\frac{\beta}{E_{s}}$ & 0 & 0 & $\frac{t}{L} \frac{1}{1-\nu^{2}}$ & 0 \\
\hline$\frac{\gamma}{E_{s}}$ & 0 & $\frac{6 I}{L^{4}}$ & $\frac{t}{L} \frac{1}{2(1+\nu)}$ & $\frac{t^{3}}{L^{3}} \frac{8\left(153-48 \nu-4 \nu^{2}\right)}{27\left(1-\nu^{2}\right)(7-2 \nu)}$ \\
\hline
\end{tabular}

Table 2: Stiffness matrix entries of the cubic topology

Equation (28) is approximate, since the overlapping volume of the nodes and adjacent edges and walls are not removed from the expression. Yet, the approximation is acceptable for the parameter ranges defined by the inequalities (27). Table 2 lists the entries of the stiffness matrix for the cubic lattice. We can observe that:

- For an open-cell lattice, the wall terms are zero, and the shear stiffness term, $\gamma$, of the edges is the smallest. Therefore, under multiaxial loading the largest material deformation is triggered by shear, which is only supported by edge bending. Hence, the cubic lattice is bending dominated.

- For a closed-cell lattice, the in-plane wall terms have all the same order of magnitude, $o\left[\frac{t}{L}\right]$. Moreover, the wall contribution becomes comparable to the edges axial contribution if $\frac{A}{L^{2}} \approx \frac{t}{L} \frac{2}{1-\nu^{2}}$; for edges with square cross section, the condition is $\frac{t}{d} \approx \frac{1-\nu^{2}}{2}\left(\frac{d}{L}\right)^{2}$. As a result, the membrane contribution of the walls becomes dominant for very small values of the wall thickness, and the lattice behaves as stretching dominated.

Figure 8a shows the design space for the stiffness matrix eigenvalues, as a fraction of the eigenvalues of the solid material. It can be observed that the shear stiffness attains very small values, that reach a ratio of $10^{-8}$. For this reason they have been omitted in Figure 8a. The smallest values correspond to the open-cell configuration, for which the lattice is bending dominated. In contrast, for a closed-cell configuration, the shear eigenvalue becomes comparable with the others. As a result, the closed cell lattice is capable of producing higher stiffness. 


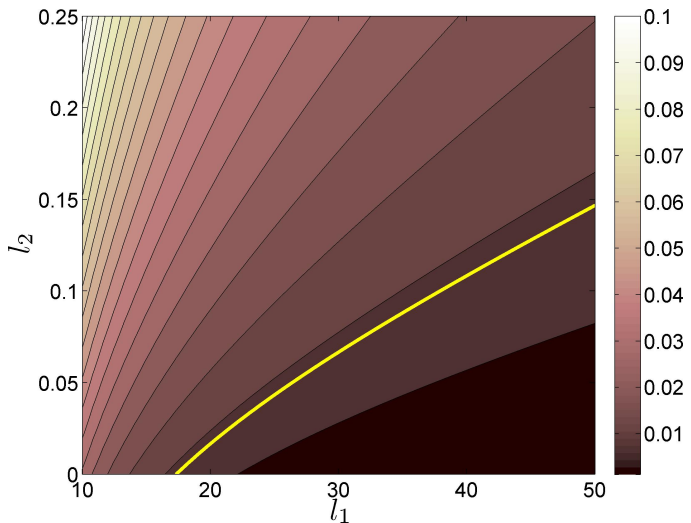

Figure 7: Cubic topology, relative density, line $\rho^{*}=0.01$ is highlighted

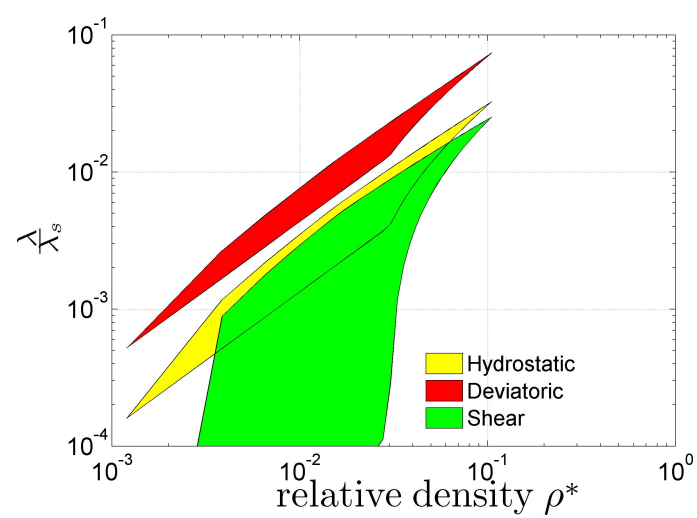

(a) Stiffness

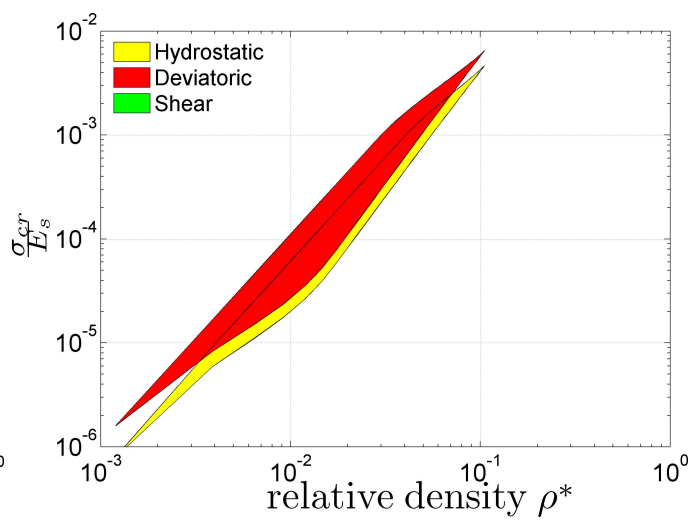

(b) Buckling strenght

Figure 8: Cubic topology, macroscopic stiffness and microscopic strength

Figure 8 and 9 report the strength charts and the von Mises surfaces for the cubic lattice. The surfaces in Figure 8 refer to the plane defined by the first deviatoric and the first shear stresses (Table 2), for a relative density of $\rho^{*}=0.01$. Each Figure reports two cases: the open cell, $l_{2}=0$, and the closed cell for the maximum wall thickness corresponding to $l_{2}=0.1467$. These charts analyse the strength of the cubic lattice in open and closed cell configurations, for different loading conditions. Figure 8 shows that the shear buckling strength of the cubic lattice is order of magnitudes smaller than the other stress states. As noted earlier, the lattice edges do not provide any effective axial contribution to load bearing; therefore the cell walls are subject to high compressive loads that produce early buckling. This is confirmed by 
the von Mises surface for buckling reported in Figure 9a, where the buckling strength of open cells cannot be defined in shear, since no compression is developed in the cell edges. For closed cell, the buckling strength is very low as it corresponds to the maximum slenderness ratio, $l_{2}=50$. The detail of the yield surface for the closed cell case is shown at the top right corner of Figure 9a.

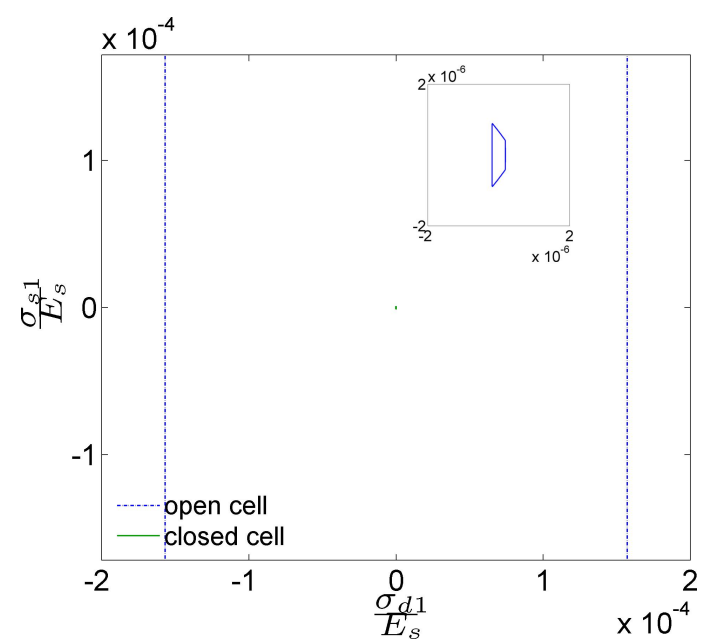

(a) Buckling

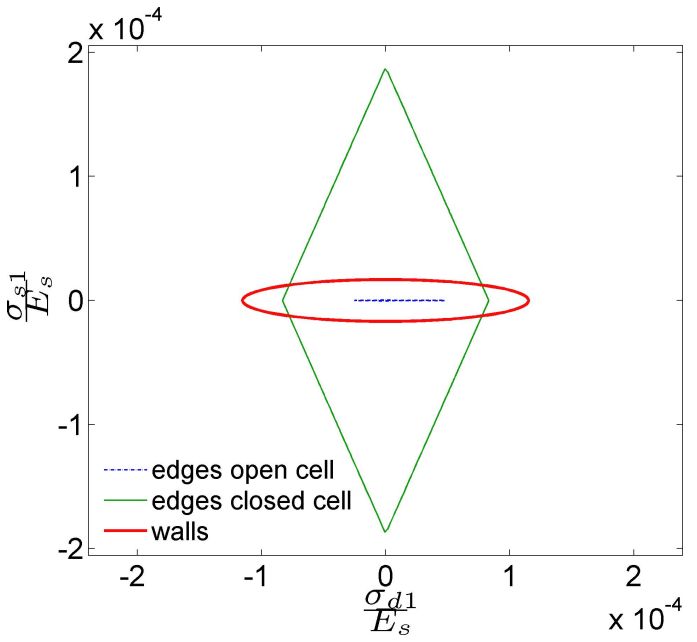

(b) Yielding

Figure 9: Cubic topology, von Mises surfaces for buckling and yielding for $\rho^{*}=10^{-2}$ and $\epsilon_{L}=0.01$

Figure 9a shows the plastic yielding von Mises surfaces for both edges and walls. As it can be observed, for the open cell case, the lattice strength is very low, being the cubic topology bending dominated. The shear strength is considerably smaller than the deviatoric strength, and it reduces to a small segment in the plot. Hence under combined loadings, even a very small shear component will inevitably cause the lattice to fail. In the closed cell case, the material limits are generally determined by the walls. As shown in Figure $9 \mathrm{~b}$, the cell walls have considerably smaller yield strength since they take most of the shear load. The higher yield load of the edges in a closed cell lattice shows that a significant strength is ensured by the lattice struts, which guarantee substantial load bearing capability after plastic yielding has occurred in the cell walls. We can thus conclude that the effect of the walls is to provide higher stiffness for the bending dominated mode. In addition, the walls provide considerably higher strength, since the shear compliance is 
very low.

\subsubsection{Face centred cube}

Figure $6 \mathrm{~b}$ shows the face centred cubic topology (FCC). Similarly to the cubic topology, the relative density can be written as

$$
\rho^{*}=3\left[(2 \sqrt{2}+1) \frac{A}{L^{2}}+\frac{t}{L}\right]
$$

The contributions to the material stiffness are reported in Table 3 . We

\begin{tabular}{|c|c|c|c|c|}
\hline & \multicolumn{2}{|c|}{ Edges } & \multicolumn{2}{c|}{ Walls } \\
\cline { 2 - 5 } & Axial & Bending & In-plane & Bending \\
\hline$\frac{\alpha}{E_{s}}$ & $\frac{(1+\sqrt{2}) A}{L^{2}}$ & $\frac{24 \sqrt{2} I}{L^{4}}$ & $\frac{2}{1-\nu^{2}} \frac{t}{L}$ & 0 \\
\hline$\frac{\beta}{E_{s}}$ & $\frac{A}{\sqrt{2} L^{2}}$ & $-\frac{12 \sqrt{2} I}{L^{4}}$ & $\frac{1}{1-\nu^{2}} \frac{t}{L}$ & 0 \\
\hline$\frac{\gamma}{E_{s}}$ & $\frac{A}{\sqrt{2} L^{2}}$ & $\frac{6(2(2+\sqrt{2}) \nu+4 \sqrt{2}+5}{4 \nu+5} \frac{I}{L^{4}}$ & $\frac{1}{2(1+\nu)} \frac{t}{L}$ & $\frac{8\left(153-48 \nu-4 \nu^{2}\right)}{27(1-\nu)(1+\nu)(7-\nu)} \frac{t^{3}}{L^{3}}$ \\
\hline
\end{tabular}

Table 3: Stiffness matrix entries of the FCC topology

can observe that, since $\frac{I}{L^{4}} \ll \frac{A}{L^{2}}$, the bending contribution to the stiffness can be neglected with respect to stretching; thus, the lattice is stretching dominated. This can be also observed in Figure 10a, where the maps of the three eigenvalues of the lattice stiffness matrix lie in the same region of the design space. Figure $10 \mathrm{~b}$ reports the relative buckling load. As it can be noted, for all relative densities, and for all stress states, the mapped regions in the material chart are quite narrow. This suggests that the cell walls have a negligible impact on the buckling strength of the FCC lattice when compared to the other topologies examined in this paper. Figure 11 maps the von Mises surfaces for buckling and yield. The buckling surfaces of the closed cell FCC and the cubic lattice are quite similar. In contrast, the buckling strength of the open cell FCC is sensibly reduced. Compared to the cubic lattice, the yield surfaces of the walls have similar shape, although the strength of the FCC is slightly reduced. This can be explained by recalling that the two cell topologies have the identical wall configurations; for the same relative density, however, the cubic topology has thicker walls, as there are no diagonal edges. With reference to the yield surfaces, the strength of the open cell case is considerably lower than its closed cell counterpart; thus the lattice walls collaborate efficiently to withstand the external loads. 


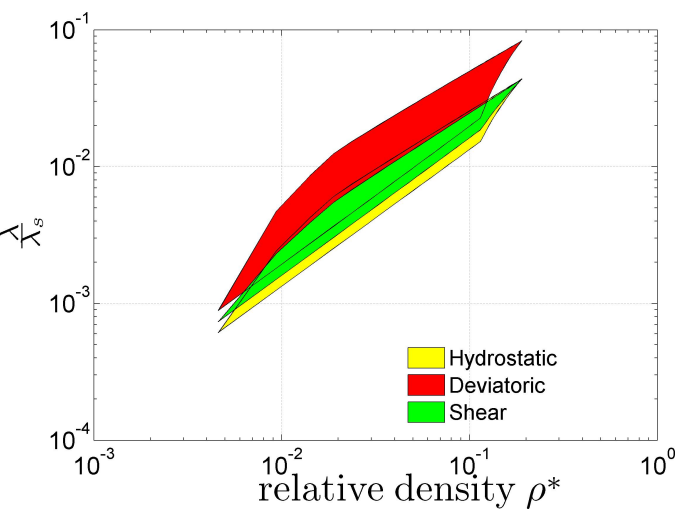

(a) Stiffness

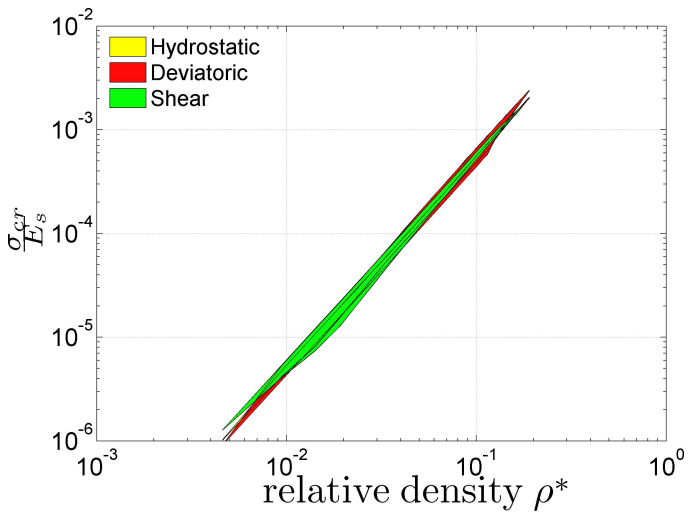

(b) Buckling strenght

Figure 10: FCC topology, macroscopic stiffness and microscopic strength

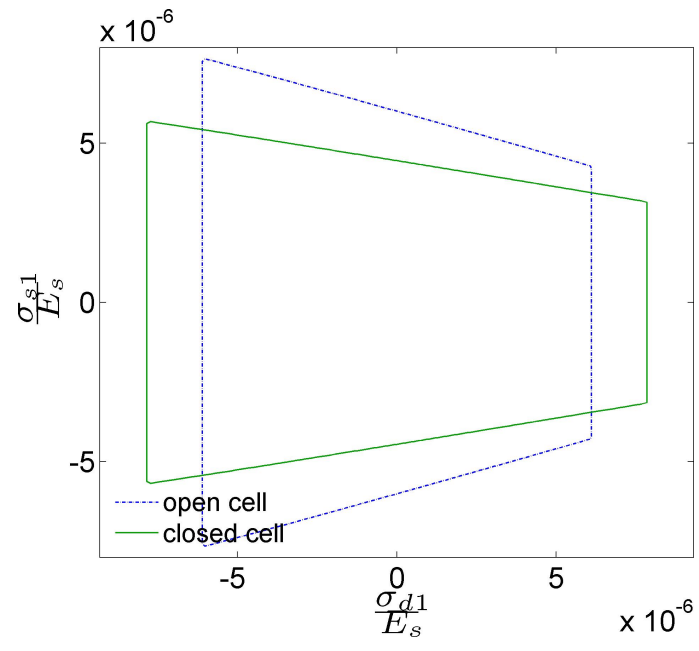

(a) Buckling

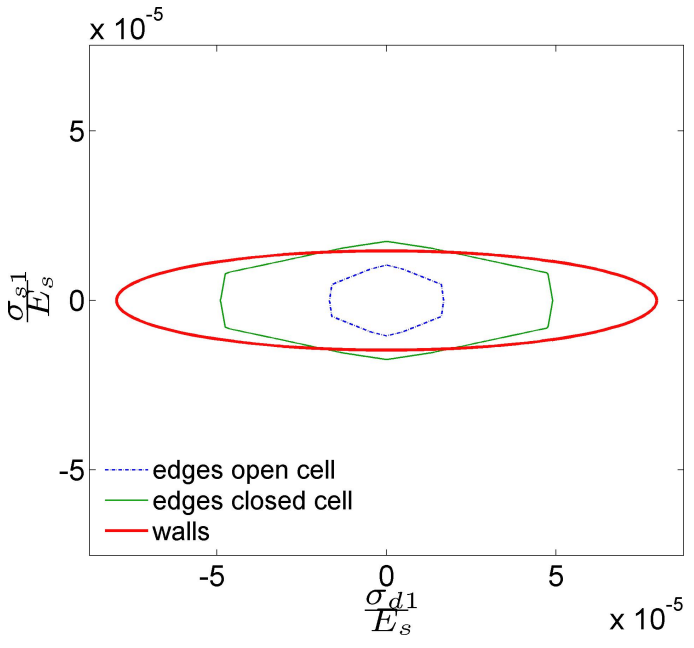

(b) Yielding

Figure 11: FCC topology, von Mises surfaces for buckling and yielding for $\rho^{*}=10^{-2}$ and $\epsilon_{L}=0.01$

\subsubsection{Body centred cube}

The Body centred cube (BCC) unit cell is shown in Figure $5 \mathrm{c}$ and in Figure 6c. The relative density of the BCC topology is given by

$$
\rho^{*}=(3+4 \sqrt{3}) \frac{A}{L^{2}}+3(1+\sqrt{2}) \frac{t}{L}
$$

Table 4 reports the terms of the material stiffness matrix. In contrast to the cubic and the FCC, all lattice elements contribute in both bending and 


\begin{tabular}{|c|c|c|c|c|}
\hline & \multicolumn{2}{|c|}{ Edges } & \multicolumn{2}{c|}{ Walls } \\
\cline { 2 - 5 } & Axial & Bending & In-plane & Bending \\
\hline$\frac{\alpha}{E_{s}}$ & $\left(1+\frac{4}{3 \sqrt{3}}\right) \frac{A}{L^{2}}$ & $\frac{128}{3 \sqrt{3}} \frac{I}{L^{4}}$ & $\frac{4+3 \sqrt{2}}{2-2 \nu^{2}} \frac{t}{L}$ & $\frac{82 \sqrt{2}}{9\left(1-\nu^{2}\right)} \frac{t^{3}}{L^{3}}$ \\
\hline$\frac{\beta}{E_{s}}$ & $\frac{4}{3 \sqrt{3}} \frac{A}{L^{2}}$ & $-\frac{64}{3 \sqrt{3}} \frac{I}{L^{4}}$ & $\frac{4(1+\sqrt{2}) \nu+\sqrt{2}}{4-4 \nu^{2}} \frac{t}{L}$ & $\frac{41 \sqrt{2}}{9\left(\nu^{2}-1\right)} \frac{t^{3}}{L^{3}}$ \\
\hline$\frac{\gamma}{E_{s}}$ & $\frac{4}{3 \sqrt{3}} \frac{A}{L^{2}}$ & $\left(6+\frac{32}{3 \sqrt{3}}\right) \frac{I}{L^{4}}$ & $\frac{3 \sqrt{2}+2-2(1+\sqrt{2}) \nu}{4\left(1-\nu^{2}\right)} \frac{t}{L}$ & $\frac{9(272+189 \sqrt{2})-6(128+81 \sqrt{2}) \nu-64 \nu^{2}}{54(7-2 \nu)\left(1-\nu^{2}\right)} \frac{t^{3}}{L^{3}}$ \\
\hline
\end{tabular}

Table 4: Stiffness matrix entries of the BCC topology

stretching to each entry of the material stiffness matrix. This suggests that the BCC topology has a better structural performance since all elements fully contribute to bear the load. This observation is confirmed by the charts in Figure 12, in which the stiffness and buckling strength for the BCC lattice are shown to be generally higher than the cubic and the FCC.

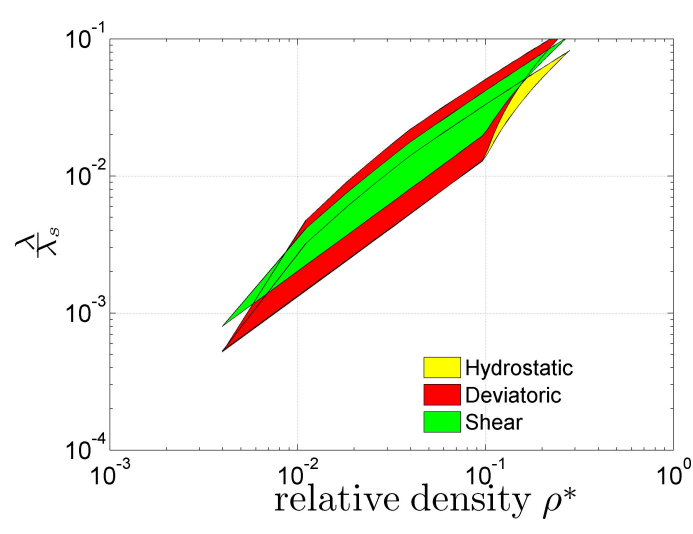

(a) Stiffness

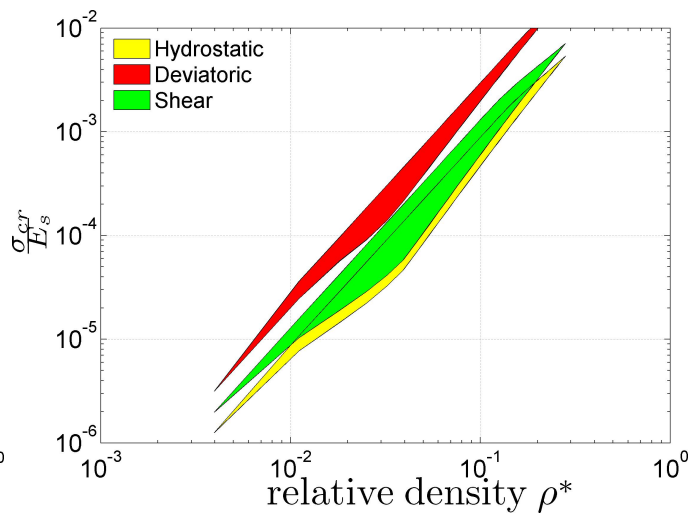

(b) Buckling strenght

Figure 12: BCC topology, macroscopic stiffness and microscopic strength

The buckling and yield surfaces for the BBC lattice are depicted in Figure 13. With reference to buckling, we observe that the BCC lattice has a lower shear strength than the deviatoric one. Figure $13 \mathrm{~b}$ gives the plastic yielding surfaces. Similarly to the other lattices, the walls generally improve the lattice strength. 


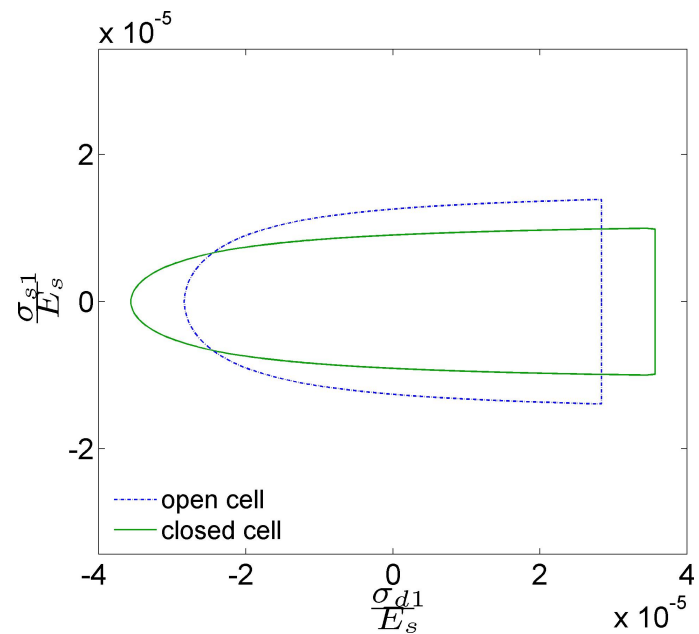

(a) Buckling

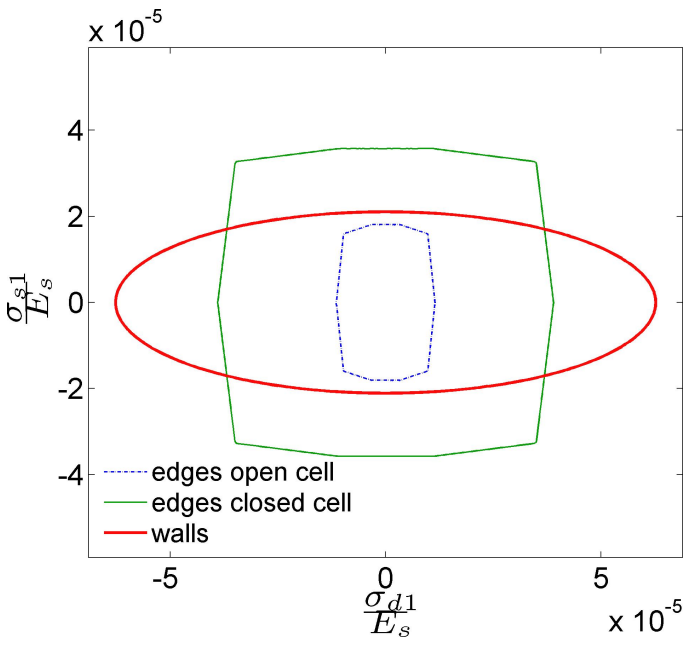

(b) Yielding

Figure 13: BCC topology, von Mises surfaces for buckling and yielding for $\rho^{*}=10^{-2}$ and $\epsilon_{L}=0.01$

\subsubsection{Regular octet}

The regular octet unit cell is shown in Figure 5d and Figure 6d. The pin-jointed open cell version of this lattice has been extensively analysed by Deshpande et al. (2001b), and Elsayed and Pasini (2010b). The results found in this paper agree with those available in the literature. Here, we present the extension to the closed cell and the rigid joint case. The relative density of the regular octet is

$$
\rho^{*}=6 \sqrt{2} \frac{A}{L^{2}}+2 \sqrt{6} \frac{t}{L}
$$

The entries of the lattice stiffness matrix are reported in Table 5. Figure 14 shows that the relative stiffness and the buckling load of the regular octet are consistently higher than the other cubic topologies for any value of relative density. In addition, the stiffness and the strength are similar to each other in each stress state. This topology is excellent for applications where both lightweight and strength are critical, since the properties of the lattice do not change significantly with the load orientation.

The von Mises stress for buckling is shown in Figure 15a for the regular octet lattice. As in the previous cases, the presence of the walls generally deteriorates the strength of the lattice. For the range of density considered here, the strength of the regular octet, for both open and closed cell, is lower than that of the $\mathrm{BCC}$; nevertheless, the properties of the octet are more 


\begin{tabular}{|c|c|c|c|c|}
\hline & \multicolumn{2}{|c|}{ Edges } & \multicolumn{2}{c|}{ Walls } \\
\cline { 2 - 5 } & Axial & Bending & In-plane & Bending \\
\hline$\frac{\alpha}{E_{s}}$ & $\frac{\sqrt{2} A}{L^{2}}$ & $\frac{12 \sqrt{2} I}{L^{4}}$ & $\frac{8 \sqrt{\frac{2}{3}}}{3-3 \nu^{2}} \frac{t}{L}$ & $-\frac{512 \sqrt{\frac{2}{3}}}{27\left(\nu^{2}-1\right)} \frac{t^{3}}{L^{3}}$ \\
\hline$\frac{\beta}{E_{s}}$ & $\frac{1}{\sqrt{2}} \frac{A}{L^{2}}$ & $-\frac{6 \sqrt{2} I}{L^{4}}$ & $-\frac{2 \sqrt{\frac{2}{3}}(3 \nu+1)}{3\left(\nu^{2}-1\right)} \frac{t}{L}$ & $\frac{256 \sqrt{\frac{2}{3}}}{27\left(\nu^{2}-1\right)} \frac{t^{3}}{L^{3}}$ \\
\hline$\frac{\gamma}{E_{s}}$ & $\frac{1}{\sqrt{2}} \frac{A}{L^{2}}$ & $\frac{6 \sqrt{2} I}{L^{4}}$ & $\frac{\sqrt{\frac{2}{3}}(3 \nu-5)}{3\left(\nu^{2}-1\right)} \frac{t}{L}$ & $-\frac{128 \sqrt{\frac{2}{3}}}{27\left(\nu^{2}-1\right)} \frac{t^{3}}{L^{3}}$ \\
\hline
\end{tabular}

Table 5: Stiffness matrix entries of the regular octet lattice

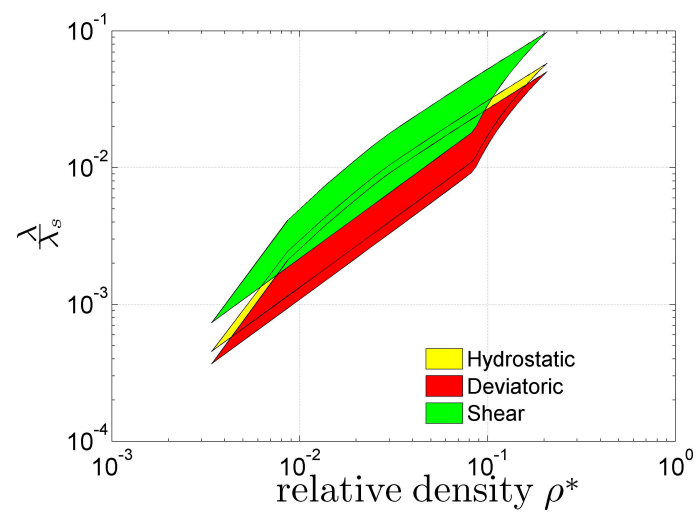

(a) Stiffness

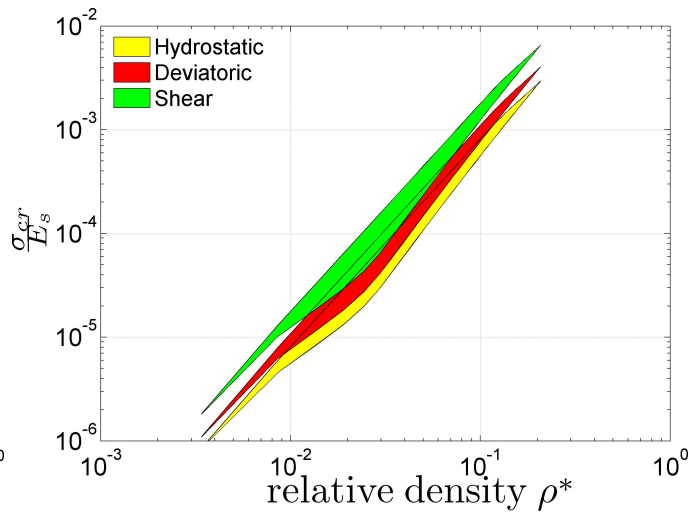

(b) Buckling strenght

Figure 14: Regular octet, macroscopic stiffness and microscopic strength

uniformly distributed than those of the BCC.

Figure 15b illustrates the yield surfaces for the regular octet. The lattice behaves almost isotropically; with respect to yield the regular octet has the best performance among the cubic lattices investigated in this paper.

\subsection{Archimedean polyhedra}

Archimedean polyhedra are a group of thirteen semi-regular convex polyhedra, whose faces are composed of different polygons. Their edges have identical vertices and equal length.

Despite of their high degree of symmetry, only a reduced set of Archimedean solid is able to produce lattices with cubic symmetry. While a group of four polyhedrons (Figure 16) cannot be tessellated, another group of four (Figure 


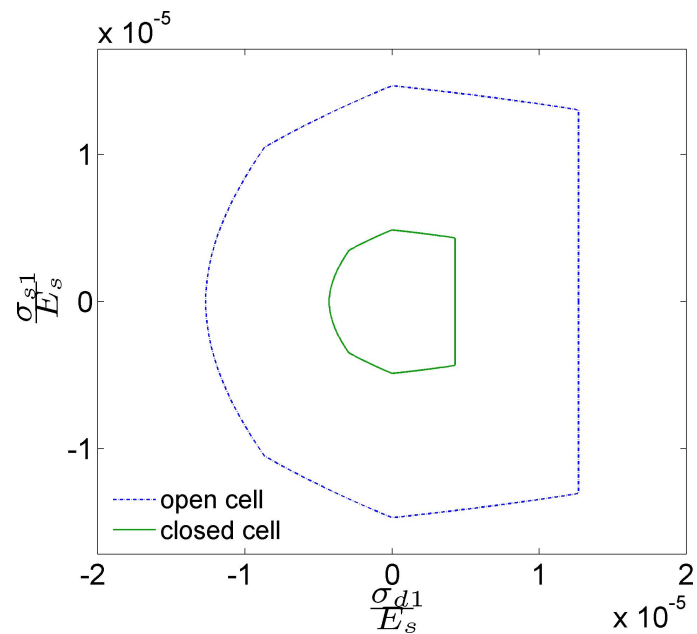

(a) Buckling

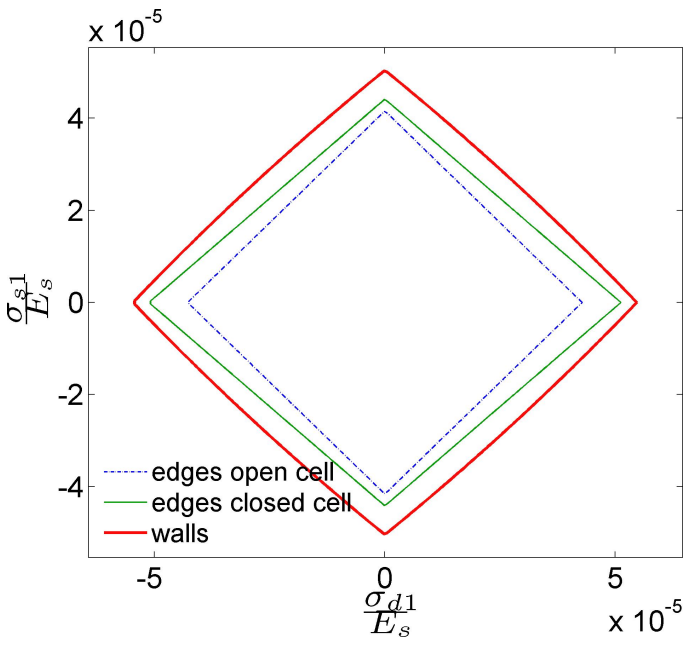

(b) Yielding

Figure 15: Von Mises surfaces of regular octet topology for buckling and yielding for $\rho^{*}=10^{-2}$ and $\epsilon_{L}=0.01$

17) is able to produce lattices, although this latter group is not examined in this paper because these lattices do not hold cubic symmetry.

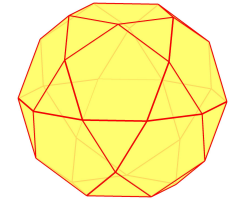

(a) Icosidodecahedron

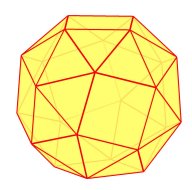

(b) Snub Cube

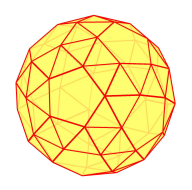

(c) Snub Dodecahedron

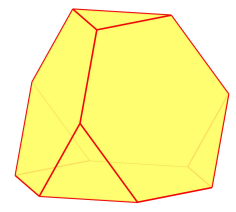

(d) Truncated Tetrahedron

Figure 16: Archimedean not tessellate-able topologies

The four polyhedra able to produce equilibrated lattices are: the Cuboctahedron (CBO), the Great Rombicuboctahedron (GRCO), the Small Rombicuboctahedron (SRCBO), the Truncated Cube (TC) and the Truncated Octahedron (TO). Table 6 lists the lattice that can be obtained by a regular tessellation of Archimedean polyhedra. Some polyhedra yield more than one lattice, depending on the tessellation directions chosen. Table 1 also lists the packing factor, $\nu^{+}$, of the lattices analysed in this paper. The packing factor is the ratio between the volume enclosed within the unit cell and the 

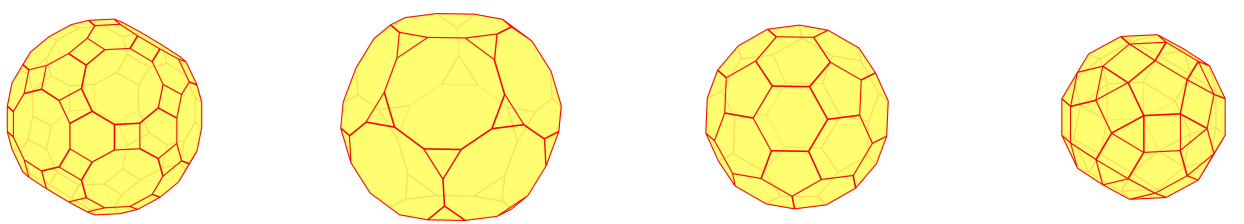

$\begin{aligned} & \text { (a) Great Rhombicosido- (b) Truncated Dodecahe- (c) Truncated } \\ & \text { decahedron }\end{aligned}$ Icosahe- (d) Small Rhombicosido-
dron

Figure 17: Archimedean not orthogonally symmetric topologies

volume defined by the tessellation vectors. As it can be noted, the Truncated Octahedron is the only polyhedron capable to tile the space without gaps. Torquato and Jiao (2009) showed that a unitary packaging factor is possible also for other arrangements of Archimedean polyhedral; yet, these tessellations do not produce cubic symmetry, and hence are disregarded here. With reference to the determinacy of the pin-jointed open cell lattices, among the lattices listed in Table 6, only the Cuboctahedron is statically determinate and has stretching dominated nature, while the others are bending dominated. The properties of the Archimedean lattices, along with the properties of the cubic lattices, are discussed in the next section.

\section{Discussion}

Bar-charts are here presented to contrast the order of magnitude of the lattice properties and comparatively discuss the lattice performance. The range of relative density and relative stiffness are plotted in Figures 18a and 18b. Among the cell topologies under investigation, the cubic based topologies can be denser than their Archimedean counterparts. With reference to the stiffness, we can observe that the highest stiffness for all the lattices corresponds to the hydrostatic macroscopic stress, for which the elements are mainly axially loaded. In contrast, the lowest stiffness generally corresponds to the pure shear macroscopic stress, for which the lattice elements are subjected to combined axial and bending loads. It can also be noted that the bending dominated lattices yield the smallest shear and deviatoric stiffness. This can be explained by recalling that bending dominated lattices under deviatoric stress respond essentially with edges and walls bending; therefore their microstructure is more compliant to these stress states.

The bar-charts in Figure 19 allow the comparison of the buckling and 


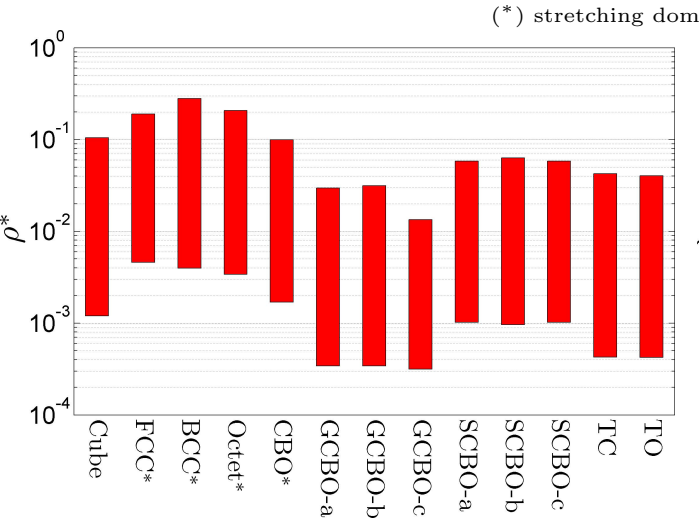

(a) Relative densities

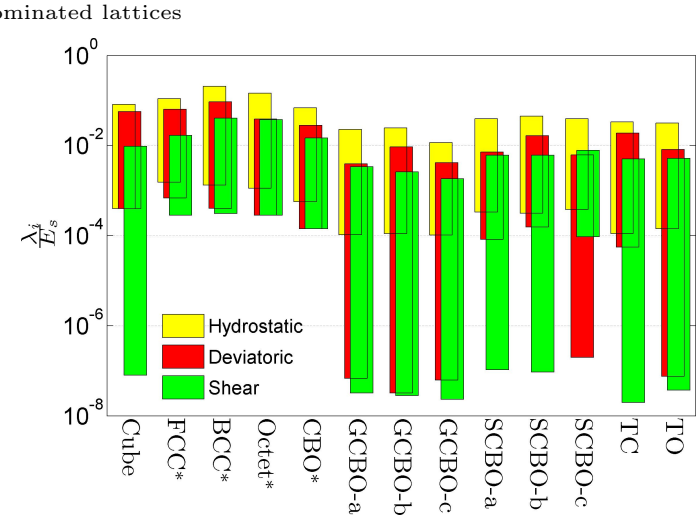

(b) Relative stiffness

yield strength. In Figure 19b, the buckling resistance was determined from the load necessary to induce buckling in both the edges and walls of the lattices. The smallest values of strength are relative to the hydrostatic stress, for which lattice elements are subject to higher normal loads. We also note that while the $\mathrm{BCC}$ and the octet have a superior buckling resistance, the latter presents more uniform yield strength for each stress state.

Figure 19b shows the yield strength of the lattices, obtained by assuming an elastic-perfect-plastic model for the solid material, with $\epsilon=0.001$. Stretching dominated lattices have higher strength than those bending dominated. Moreover, since the buckling and yield strength of stretching dominated topologies exhibit ranges which are comparable for different stress conditions, the strength of a stretching dominated lattice does not depends on the directions of the applied loads.

Figure 20 shows the charts for the shear eigenvalue. We observe that the lattice properties cluster into different regions. The stretching dominated lattices are lighter and stiffer with reduced property ranges, as opposed to the bending dominated. We also note that the stiffness of stretching dominated lattices scales with the first power of the relative density, as illustrated by the top and bottom boundaries of the property regions in Figure 20a. In contrast, with reference to bending dominated lattices, the stiffness of the open cell configurations, which occupy the lower border of chart 20b, scales with $\rho^{*^{2}}$. For closed cell configurations, on the other hand, are located at the upper boundary; their stiffness scales with the first power of $\rho^{*}$, typi- 


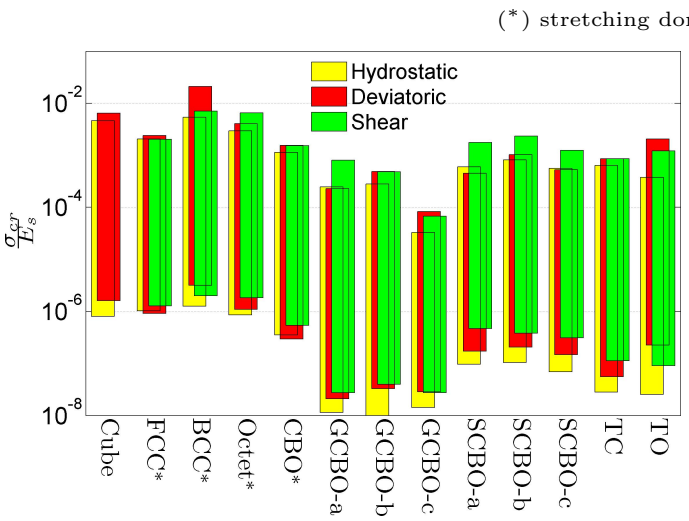

(a) Buckling strength

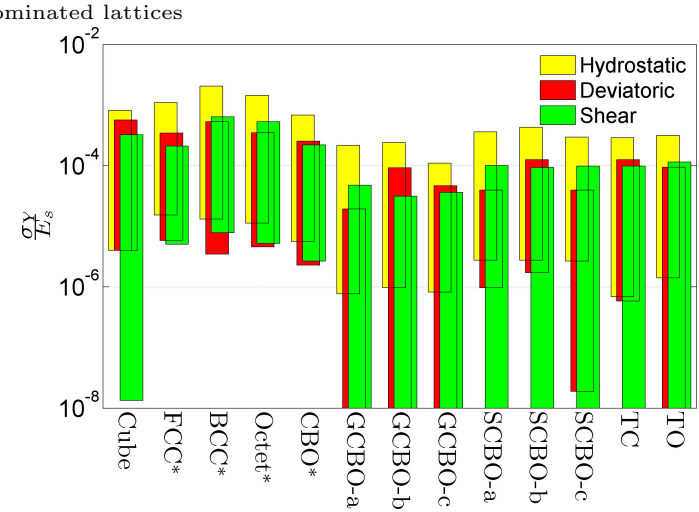

(b) Yield strength

Figure 19: Lattice strength

cal of a stretching dominated behaviour. Furthermore, bending dominated lattices with closed cell have stiffness similar to the stretching dominated. These observations confirm what already noted for the cubic lattice. The effect of the walls is to induce a stretching dominated behaviour in those topologies that are bending dominated in the open cell configuration. Figure

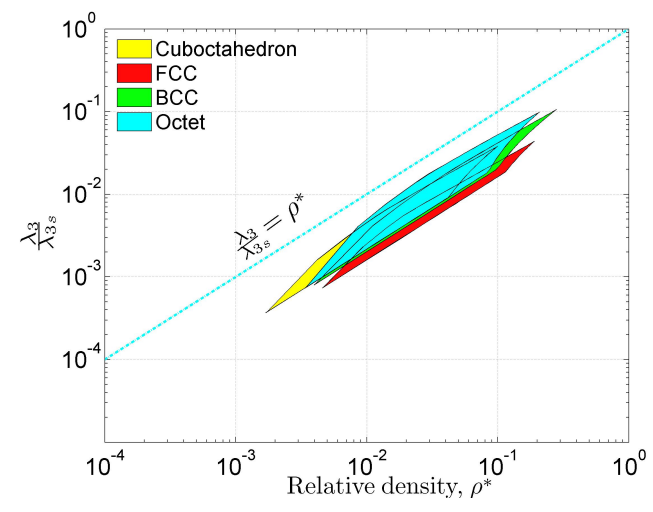

(a) Stretching dominated lattices

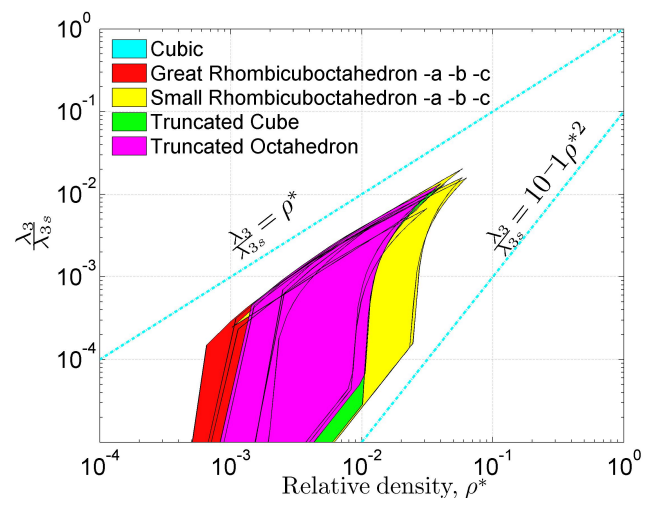

(b) Bending dominated lattices

Figure 20: Lattice stiffness for macroscopic shear stress

21 illustrates the buckling strength for a shear macroscopic stress. In this case, both the classes of lattices, with either open or closed cells, scale with the second power of the density. One exception is the open cell cubic lattice, which cannot withstand buckling. Furthermore, although the stretching 
dominated lattices generally possess higher strength, the bending dominated ones are generally stronger for medium to low ranges of density.

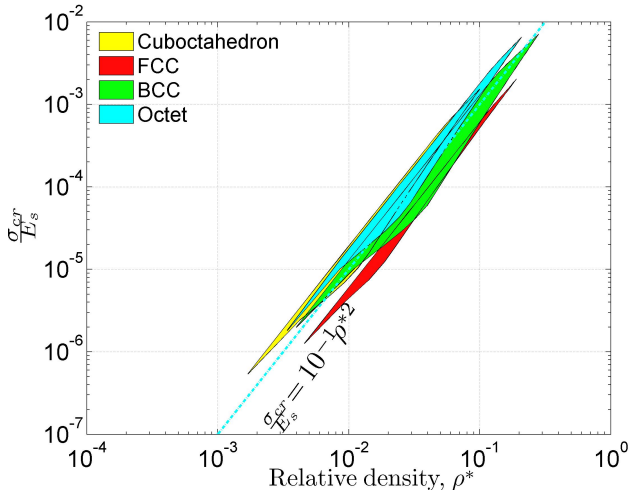

(a) Stretching dominated lattices

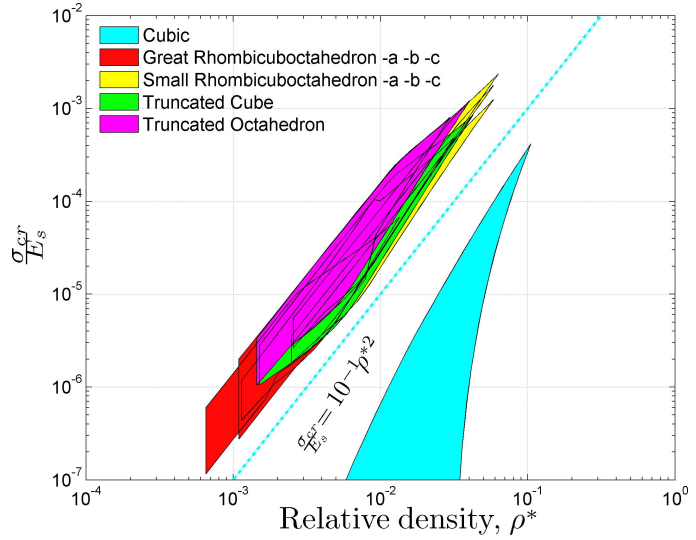

(b) Bending dominated lattices

Figure 21: Buckling strength for macroscopic shear stress

Figure 22 shows the yield charts for shear stress. As in the charts for stiffness, closed cell lattices always behave as stretching dominated, as opposed to open cell. With respect to the maximum achievable strength, stretching dominated lattices are stronger, although the properties overlap at the intersection between the lower bound of the stretching dominated lattices and the upper bound of the bending dominated ones.

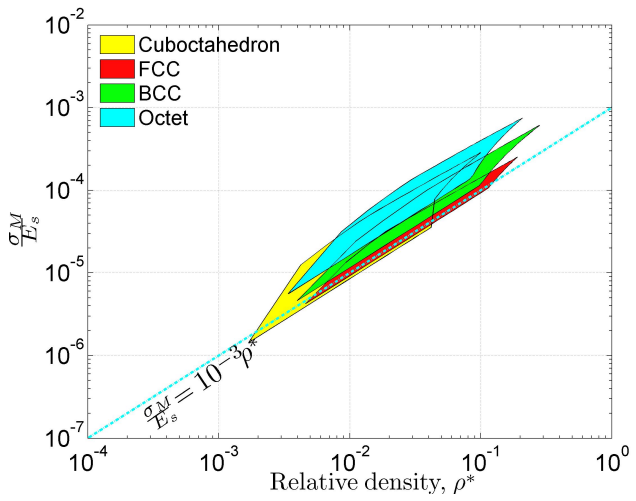

(a) Stretching dominated lattices

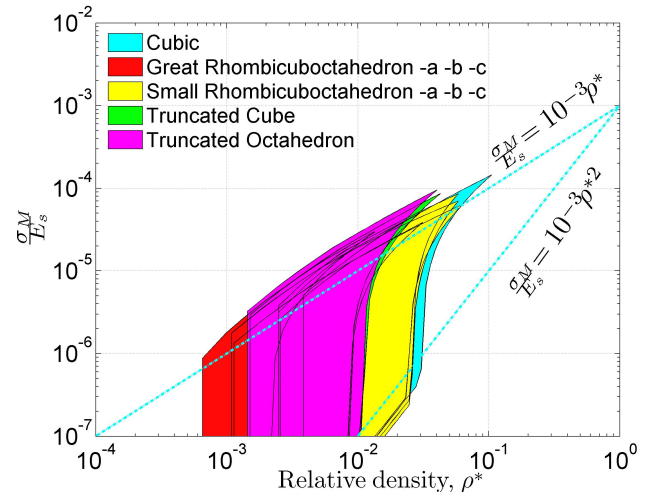

(b) Bending dominated lattices

Figure 22: Yielding strength for shear macroscopic stress 
In Figure 23, the lattice buckling strength is plotted against the relative stiffness for shear stress. A distinct separation exists among the two lattice classes. The stretching dominated can reach higher stiffness and strength, and map a reduced area of the design space. In contrast, the bending dominated lattices span a larger area with reduced strength and stiffness.

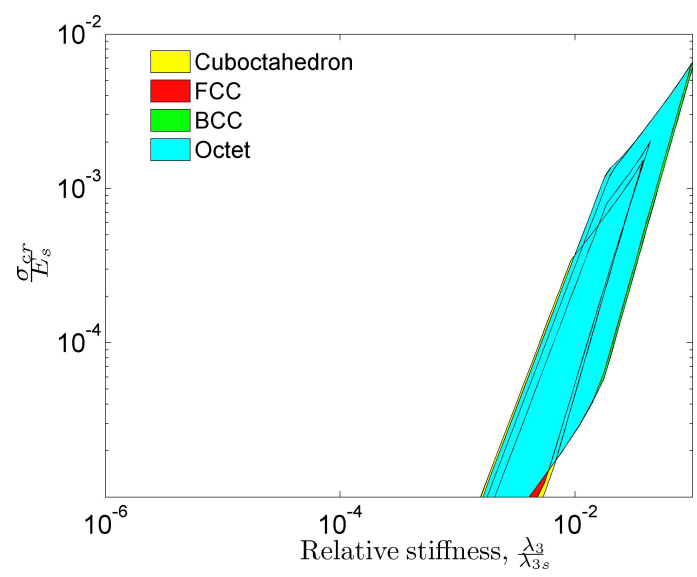

(a) Stretching dominated lattices

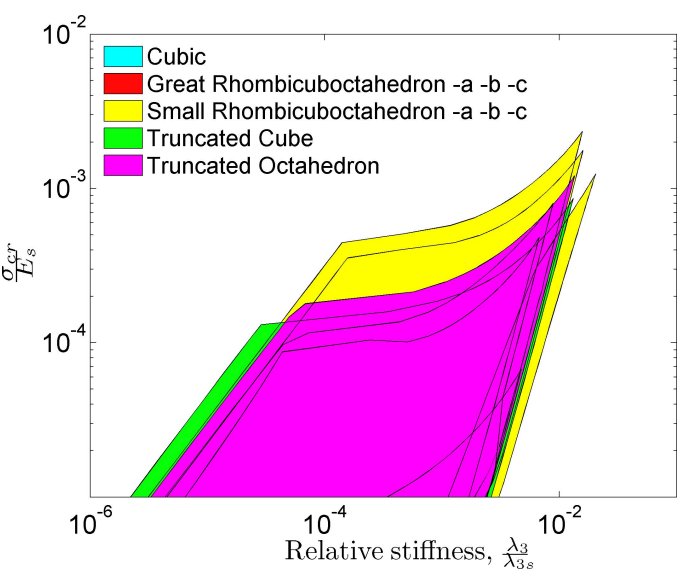

(b) Bending dominated lattices

Figure 23: Buckling strength vs relative stiffness for macroscopic shear stress

From the above charts, we draw an important remark. Lattices with bending dominated behaviour in open cell configuration behave as stretching dominated in closed cell configuration. As shown in more detail for the cubic lattice, the in-plane stiffness of the walls in closed-cell lattices reduces the bending load on the edges, and ensures stretching dominated behaviour. We also note that the highest values of stiffness and strength are reached by the open cell stretching dominated lattices, while the properties of bending dominated lattices span over larger ranges. Hence for applications requiring high compliance, the bending dominated topologies are better suited than the stretching dominated ones, which are more suitable for applications requiring high strength and low weight.

It can also be noticed that the property ranges of bending and stretching dominated lattices are generally distinct and cover complementary regions in the material charts; they overlap only in limited cases. Some topologies have a larger span of properties with respect to others; however, none of them can envelope all.

The multiscale procedure and the charts presented in this paper can be 
used to optimize the macroscopic stiffness of a complex component made of lattice material and to assess its buckling and yield strength. The method can be readily automated and integrated with optimization algorithms for optimal material design. The charts, presented in the paper confirm how critical the choice of a cell topology might be. They are general since the properties are normalized for a given solid material. When material selection is coupled to topology selection and lattice geometric parameters optimization, a large variety of mechanical behaviours can be obtained to satisfy different design requirements and conflicting objective functions (Vigliotti and Pasini, 2011).

\section{Conclusions}

A multiscale procedure for the analysis of three-dimensional open and closed cell lattices has been presented. The procedure allows the macro and microscale analysis of unit cells with arbitrary topology with both pin and rigid joints. At the macroscale, the lattice stiffness is determined by modelling the discrete lattice as a continuous medium. At the microscale, the stress induced by a macroscopic stress field in each cell element is assessed to verify the occurrence of yield and buckling failure. Buckling load and modes have been also determined.

The procedure has been applied to the analysis of four cubic-based lattices and nine lattices based on Archimedean polyhedra. For open and closed cell topologies, the influence of edges cross section and wall thickness has been quantified. The stiffness and strength properties have been plotted on material charts. While the results found in this paper for the pin-jointed regular octet and the truncated dodecahedron are consistent to those found in literature (Zhu et al., 1997; Deshpande et al., 2001b; Wallach and Gibson, 2001; Gong et al., 2005), the properties of the other cell topologies have been provided here for the first time. The results show that wall in-plane forces effectively contribute to the stiffness and yield strength of the lattice; hence if the walls are present, open cell topologies that are bending dominated behave as stretching dominated.

The study described in this paper is limited to a linear analysis of lattice properties. Geometric non linearities, however, have a considerable impact on the effective behaviour of a lattice, especially for bending dominated topologies, and they will be the subject of future studies. 


\section{Appendix A. Derivation of the lattice topology matrices}

This section describes the derivation of the property matrices of the Body Centred Cubic topology, which is here selected as a paradigm cell (Figure A.24) to demonstrate the procedure. The matrices are necessary to find the expression for the specific strain energy and macroscopic stiffness matrix of the lattice. The following analysis is general and can be applied to any arbitrary topology.

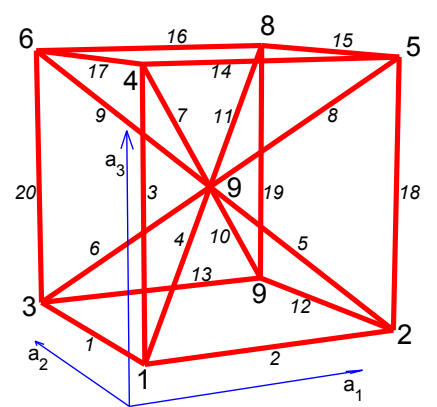

(a) unit cell

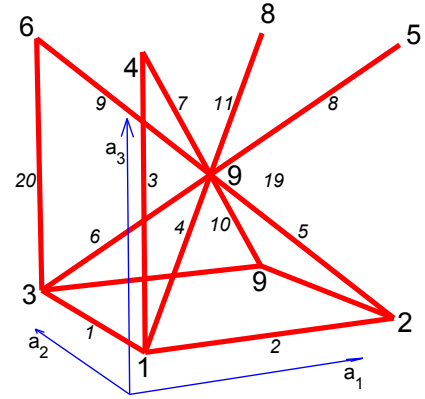

(b) primitive cells

Figure A.24: BCC nodes and edges numbering

To obtain the deformation work, it is necessary to identify the primitive cell that is used to tessellate the space and generate the lattice. With this purpose, the duplicated elements that are corresponding along any periodic direction have to be removed. Figure A.24 shows both the unit cell and the primitive cell, obtained by removing the edges from 12 to 19 . We start considering the matrices $\mathbf{B}_{0}$ and $\mathbf{B}_{a}$ of equation (7), which expresses, in a concise form, the DoF of all the nodes of the unit cell as a function of the DoFs of the independent nodes, and of the change in the periodic vectors. As shown in Figure A.24, the BCC topology includes only one internal node, node 9, and eight boundary nodes. According to equation (5), the components of the displacement of the node 4 can be expressed as

$$
\begin{aligned}
u_{4} & =u_{1}+\Delta a_{3 x} \\
v_{4} & =v_{1}+\Delta a_{3 y} \\
w_{4} & =w_{1}+\Delta a_{3 z}
\end{aligned}
$$

where the components of the displacements are defined in as shown in Figure 3. Introducing the array $\mathbf{d}_{i}=\left[u_{i}, v_{i}, w_{i}, \theta_{x i}, \theta_{y i}, \theta_{z i}\right]^{T}$, and collecting all DoFs 
of the node allows to write in compact form

$$
\mathbf{d}_{4}=\mathbf{d}_{1}+\mathbf{I}_{t} \boldsymbol{\Delta} \mathbf{a}_{3} \quad \text { with } \quad \mathbf{I}_{t}=\left[\begin{array}{lll}
1 & 0 & 0 \\
0 & 1 & 0 \\
0 & 0 & 1 \\
0 & 0 & 0 \\
0 & 0 & 0 \\
0 & 0 & 0
\end{array}\right]
$$

We remark that in equation (A.2) we assume that a change in the periodic vectors directly produces only relative displacements of the boundary nodes, whereas the rotational DoFs are determined by imposing the periodic equilibrium conditions.

As it can be verified, the DoFs of all the nodes of the BCC topology can be expressed as a function of the DoFs of nodes 1 and 9, and of the change in the periodic vectors as follows

$$
\begin{array}{ll}
\mathbf{d}_{2}=\mathbf{d}_{1}+\mathbf{I}_{t} \Delta \mathbf{a}_{1} & \mathbf{d}_{3}=\mathbf{d}_{1}+\mathbf{I}_{t} \Delta \mathbf{a}_{2} \\
\mathbf{d}_{4}=\mathbf{d}_{1}+\mathbf{I}_{t} \Delta \mathbf{a}_{3} & \mathbf{d}_{5}=\mathbf{d}_{1}+\mathbf{I}_{t} \Delta \mathbf{a}_{1}+\mathbf{I}_{t} \Delta \mathbf{a}_{3} \\
\mathbf{d}_{6}=\mathbf{d}_{1}+\mathbf{I}_{t} \Delta \mathbf{a}_{2}+\mathbf{I}_{t} \Delta \mathbf{a}_{3} & \mathbf{d}_{7}=\mathbf{d}_{1}+\mathbf{I}_{t} \Delta \mathbf{a}_{1}+\mathbf{I}_{t} \Delta \mathbf{a}_{2} \\
\mathbf{d}_{8}=\mathbf{d}_{1}+\mathbf{I}_{t} \boldsymbol{\Delta} \mathbf{a}_{1}+\mathbf{I}_{t} \Delta \mathbf{a}_{2}+\mathbf{I}_{t} \Delta \mathbf{a}_{3} &
\end{array}
$$

Introducing the block matrices, we obtain the equation (7), that we rewrite here as

$$
\mathbf{d}=\mathbf{B}_{0} \mathbf{d}_{0}+\mathbf{B}_{\mathrm{a}} \Delta \mathbf{a}
$$

where the following positions hold

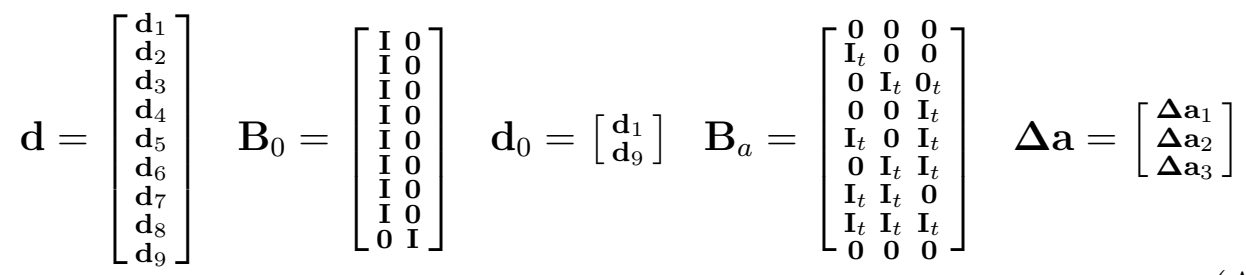

$\mathbf{d}$ is a block array collecting the DoFs of all the nodes of a unit cell.

$\mathbf{B}_{0}$ expresses the dependencies among the nodes of the unit cell in relation to the periodic vectors; it has as many row blocks as the number of nodes of the unit cell and as many column blocks as the numbers of independent nodes; for each row only one column block is non zero; the blocks are identity matrices of the size of the number of DoFs for each node.

$\mathbf{d}_{0}$ is an array collecting the DoFs of the independent nodes.

$\mathbf{B}_{a}$ expresses the relative displacement between corresponding boundary nodes of the unit cells, as a function of the change in the periodic vectors; 
it has as many row blocks as the number of nodes of the unit cell; it has also as many column blocks as the number of independent periodic vectors; the blocks are signed to take into account negative translations.

$\Delta \mathbf{a}$ is an array collecting the change of the periodic vectors.

We will now consider the expression of the periodic equilibrium condition and matrix $\mathbf{A}_{0}$, in equation (9). Under the action of a macroscopic stress, each cell is subjected to the action of the surrounding cells. Due to periodicity, the internal forces in the elements that are corresponding along the translational vectors must be equal; thus we can express the action of the surrounding cells in terms of the internal forces of a single primitive cell. The equilibrium problem of the infinite lattice is finally reduced to the equilibrium of a single cell.

Let us consider node 2 in the BCC primitive cell (Figure A.24). The edges connecting at node 2 from the surrounding cells are: (i) edges 1, 2 and 3 , through the vector $\mathbf{a}_{1}$, whose internal force sum is the force in node 1 ; (ii) edges 3 and 7 , through $\mathbf{a}_{1}-\mathbf{a}_{3}$, whose sum of internal force is the force in node 4; (iii) edges 1 and 6 , through $\mathbf{a}_{1}-\mathbf{a}_{2}$, whose sum of internal forces is the force at node 3; (iv) edge 10, which connects on node 2 through a translation along the vector $-\mathbf{a}_{2}$, whose internal force is equal to the force in node 7. Summarizing the periodic equilibrium condition for node 2 is the following

$$
\mathbf{F}_{1}+\mathbf{F}_{2}+\mathbf{F}_{3}+\mathbf{F}_{4}+\mathbf{F}_{5}+\mathbf{F}_{6}+\mathbf{F}_{7}+\mathbf{F}_{8}=\mathbf{0}
$$

It can be easily verified that the same equation is obtained, if the procedure is applied to all boundary nodes. For node 9, which is internal and only connect edges of the same cell, the equilibrium condition is simply: $\mathbf{F}_{9}=\mathbf{0}$. In general, the periodic equilibrium conditions for the primitive cell can be expressed as follows: (i) for the internal nodes, the nodal forces must be zero since the internal nodes connect only elements of the unit cell, and no body force is applied; (ii) for the boundary nodes, the sum of the nodal forces of all the nodes that are corresponding along the periodic vectors must be zero. In matrix form, the periodic equilibrium condition can be expressed by equation (9), which here reported

$$
\mathbf{A}_{0} \mathbf{F}=\mathbf{A}_{0} \mathbf{K}_{\mathrm{uc}} \mathbf{d}=\mathbf{0}
$$

where $\mathbf{A}_{0}$ is a block matrix assembled as follows. Each block is a square matrix and its dimension is equal to the number of DoFs of each node. $\mathbf{A}_{0}$ 
has as many row blocks as the number of independent nodes in the primitive cell, and as many column blocks as the total number of nodes of the unit cell. Each block row corresponds to a single independent node. For each internal node, all column blocks entries are zero except for an identity block corresponding to the DoFs of the node; on the other hand for the boundary nodes, all column blocks corresponding to the DoFs of the nodes belonging to the same class are identity matrices, the others are zero.

\section{References}

\section{References}

Asaro, R. J., Lubarda, V. A., 2006. Mechanics of solids and materials. Cambridge University Press, Cambridge.

Ashby, M., 2011. Hybrid materials to expand the boundaries of materialproperty space. Journal of the American Ceramic Society 94, S3-S14.

Ashby, M. F., 2005. Materials Selection in Mechanical Design, 3rd Edition. Elsevier.

Banhart, J., 2001. Manufacture, characterisation and application of cellular metals and metal foams. Progress in Materials Science 46 (6), 559-U3.

Bazeley, G., Cheung, Y., Irons, B., O.C., Z., 1965. Triangular elements in plate bending-conforming and non-conforming solutions. Proc. Conf. on Matrix Methods in Structural Mechanics, WPAFB, Ohio, 547-576.

Bendsoe, M. P., Kikuchi, N., 1988. Generating optimal topologies in structural design using a homogenization method. Computer Methods in Applied Mechanics and Engineering 71 (2), 197-224, r0985 Times Cited:1174 Cited References Count:57.

Bidanda, B., Bartolo, P., 2008. Virtual Prototyping \& Bio Manufacturing in Medical Applications. Engineering. Springer US.

Cook, R. D., Malkus, D. S., Plesha, M. E., 1989. Concepts and Applications of Finite Element Analysis, 3rd Edition. John Wiley and Sons Canada, Ltd. 
Deshpande, V. S., Ashby, M. F., Fleck, N. A., 2001a. Foam topology: Bending versus stretching dominated architectures. Acta Materialia 49 (6), $1035-1040$.

Deshpande, V. S., Fleck, N. A., Ashby, M. F., 2001b. Effective properties of the octet-truss lattice material. Journal of the Mechanics and Physics of Solids 49 (8), 1747-1769.

Elsayed, M. S. A., Pasini, D., 2010a. Analysis of the elastostatic specific stiffness of $2 \mathrm{~d}$ stretching-dominated lattice materials. Mechanics of Materials $42(7), 709-725$.

Elsayed, M. S. A., Pasini, D., 2010b. Multiscale structural design of columns made of regular octet-truss lattice material. International Journal of Solids and Structures 47 (14-15), 1764-1774.

Elsayed, M.S.A., P. D., 2010. Characterization and performance optimization of $2 \mathrm{~d}$ lattice materials with hexagonal bravais lattice symmetry. Vol. 5. pp. 1315-1323, proceedings of the ASME International Design Engineering Technical Conferences and Computers and Information in Engineering Conference 2009, DETC2009.

Fleck, N.A., D. V. A. M., 2010. Micro-architectured materials: Past, present and future. Proceedings of the Royal Society A: Mathematical, Physical and Engineering Sciences 466 (2121), 2495-2516, cited By (since 1996) 0.

Gibson, L. J., Ashby, M. F., Schajer, G. S., Robertson, C. I., 1982. The mechanics of two-dimensional cellular materials. Proceedings of the Royal Society of London Series a-Mathematical Physical and Engineering Sciences 382 (1782), 25-42.

Gonella, S., Ruzzene, M., 2008. Homogenization and equivalent in-plane properties of two-dimensional periodic lattices. International Journal of Solids and Structures 45 (10), 2897-2915.

Gonella, S., Ruzzene, M., 2010. Multicell homogenization of one-dimensional periodic structures. Journal of Vibration and Acoustics, Transactions of the ASME 132 (1), 0110031-01100311. 
Gong, L., Kyriakides, S., Jang, W. Y., 2005. Compressive response of opencell foams. part i: Morphology and elastic properties. International Journal of Solids and Structures 42 (5-6), 1355-1379.

Hassani, B., Hinton, E., 1998. A review of homogenization and topology optimization i - homogenization theory for media with periodic structure. Computers \& Structures 69 (6), 707-717.

Hutchinson, R. G., Fleck, N. A., 2006. The structural performance of the periodic truss. Journal of the Mechanics and Physics of Solids 54 (4), 756782 .

Kouznetsova, V., Geers, M. G. D., Brekelmans, W. A. M., 2002. Multiscale constitutive modelling of heterogeneous materials with a gradientenhanced computational homogenization scheme. International Journal for Numerical Methods in Engineering 54 (8), 1235-1260.

Kumar, R. S., McDowell, D. L., 2004. Generalized continuum modeling of 2-d periodic cellular solids. International Journal of Solids and Structures 41 (26), 7399-7422.

Langley, R. S., 1996. The response of two-dimensional periodic structures to point harmonic forcing. Journal of Sound and Vibration 197 (4), 447-469, cited By (since 1996): 47.

Lombardo, M., Askes, H., 2011. Higher-order gradient continuum modelling of periodic lattice materials. Computational Materials ScienceArticle in Press.

Noor, A. K., 1988. Continuum modeling for repetitive lattice structures. Applied Mechanics Reviews 41 (7), 285-296.

Ohno, N., Takahashi, Y., Okumura, D., 2010. Yield and buckling behavior of kelvin open-cell foams subjected to uniaxial compression. International Journal of Mechanical Sciences 52 (2), 377-385.

Pasini, D., 2007. Shape transformers for material and shape selection of lightweight beams. Materials \& Design 28 (7), 2071-2079.

Phani, A. S., W. J. F. N., 2006. Wave propagation in two-dimensional periodic lattices. Journal of the Acoustical Society of America 119 (4), 19952005 . 
Ramirez, D.A., M. L. L. S. T. Y. M. E. M. J. M. B. G. S. M. F. W. R., 2011. Open-cellular copper structures fabricated by additive manufacturing using electron beam melting. Materials Science and Engineering A 528 (16-17), 5379-5386.

Strang, G., 2006. Linear Algebra and Its Applications, 4th Edition. Brooks Cole.

Suiker, A. S. J., Metrikine, A. V., de Borst, R., 2001. Comparison of wave propagation characteristics of the cosserat continuum model and corresponding discrete lattice models. International Journal of Solids and Structures 38 (9), 1563-1583.

Torquato, S., Jiao, Y., 2009. Dense packings of the platonic and archimedean solids. Nature 460 (7257), 876-879, 10.1038/nature08239.

Vigliotti, A., Pasini, D., 2011. Structural optimization of lattice materials. Proceedings of the ASME 2011 International Design Engineering Technical Conferences \& Computers and Information in Engineering Conference, IDETC/CIE 2011, August 28-31, 2011, Washington, DC, USA.

Vigliotti, A., Pasini, D., 2012. Linear multiscale analysis and finite element validation of stretching and bending dominated lattice materials. Mechanics of Materials 46, 57-68.

Wallach, J. C., Gibson, L. J., 2001. Mechanical behavior of a threedimensional truss material. International Journal of Solids and Structures 38 (40-41), 7181-7196.

Wang, A. J., McDowell, D. L., 2004. In-plane stiffness and yield strength of periodic metal honeycombs. Journal of Engineering Materials and Technology-Transactions of the Asme 126 (2), 137-156.

Warren, W. E., Kraynik, A. M., 1987. Foam mechanics - the linear elastic response of two-dimensional spatially periodic cellular materials. Mechanics of Materials 6 (1), 27-37.

Zhu, H. X., Knott, J. F., Mills, N. J., 1997. Analysis of the elastic properties of open-cell foams with tetrakaidecahedral cells. Journal of the Mechanics and Physics of Solids 45 (3), 319-. 
Zienkiewicz, O., Taylor, R. L., 2005. The Finite Element Method for Solid and Structural Mechanics, 6th Edition. Elsevier. 
Table 6: Archimedean topologies 